\title{
Theory of itinerant-electron ferromagnetism
}

\author{
Fusayoshi J. Ohkawa \\ Division of Physics, Graduate School of Science, Hokkaido University, Sapporo 060-0810, Japan
}

(Received:

\begin{abstract}
A theory of Kondo lattices or a $1 / d$ expansion theory, with $d$ spatial dimensionality, is applied to studying itinerant-electron ferromagnetism. Two relevant multi-band models are examined: a band-edge model where the chemical potential is at one of band-edges, the top or bottom of bands, and a flat-band model where one of bands is almost flat or dispersionless and the chemical potential is at the flat band. In both the models, a novel ferromagnetic exchange interaction arises from the virtual exchange of pair excitations of quasiparticles; it has two novel properties such as its strength is in proportion to the effective Fermi energy of quasiparticles and its temperature dependence is responsible for the Curie-Weiss law. When the Hund coupling $J$ is strong enough, the superexchange interaction, which arises from the virtual exchange of pair excitations of electrons across the MottHubbard gap, is ferromagnetic. In particular, it is definitely ferromagnetic for any nonzero $J>0$ in the large limit of band multiplicity. Ferromagnetic instability occurs, when the sum of the two exchange interactions is ferromagnetic and it overcomes the quenching of magnetic moments by the Kondo effect or local quantum spin fluctuations and the suppression of magnetic instability by the mode-mode coupling among intersite spin fluctuations.
\end{abstract}

75.10.-b, 71.10.-w, 75.30.Et, 75.10.Lp

\section{INTRODUCTION}

Itinerant-electron ferromagnetism has been a long standing and important issue since the advent of quantum mechanics. When the Hubbard model, for example, is considered in the Hartree-Fock approximation, a ferromagnetic instability condition is given by

$$
U \rho_{0}(0)>1
$$

with $U$ the on-site repulsion and $\rho_{0}(0)$ the unrenormalized density of states at the chemical potential. Local correlations between electrons with parallel spins are accurately treated in any approximation as long as the Pauli principle is taken into account. In the Hartree-Fock approximation, however, no local correlations between electrons with antiparallel spins are taken into account; paramagnetic states are disfavored so that magnetic states are relatively favored. The condition $(1.1)$ is never reliable. Local spin correlations should be accurately taken into account far beyond the Hartree-Fock approximation in any reliable theory.

In 1963, Kanamori 1 studied ferromagnetism in $\mathrm{Ni}$ in the $T$-matrix approximation, which is relevant for low densities of electrons or holes. He showed that a ferromagnetic state is stabilized not only when Eq. (1.1) is satisfied but also when the density of states has a sharp peak within broad bands and the chemical potential is at the peak. Mielke2, Mielke and Tasaki3, Tasakit, and Kusakabe and Aokis showed that the ground state of certain multi-band models is ferromagnetic. In their models, one of bands is dispersionless or flat and the density of states is similar to that of Kanamori's. These theories show that the existence of a flat band or flat bands is one of the most relevant conditions for itinerant-electron ferromagnetism. In many ferromagnets such as $\mathrm{Ni}$, the density of electrons or holes is low and the chemical potential is at one of the band edges. One may argue that this condition must also be relevant. Call these two conditions flat-band and band-edge conditions in this paper.

The ground state can be ferromagnetif even if neither of these conditions is satisfied. Nagaokal and Thouless showed that when a single hole is introduced into the just half-filled system or the electron number is $N-1$, with $N$ the number of lattice sites, the ground state is a completely polarized ferromagnetic state in the limit of $U \rho_{0}(0) \rightarrow+\infty$. Because spin waves of this state are abnormal and any extension of their arguments to low but nonzero densities of holes is difficult, it is doubtful if a ferromagnetic state is stabilized in the thermodynamic limit of small hole densities where the number of electrons is $N(1-\delta)$, with $N \rightarrow+\infty$ and $\delta \rightarrow+0$.

In 1963, another two distinguished papers 80 were published. Hubbard 8 showed that a band splits into two bands, the lower and upper Hubbard bands. When the density of states of unrenormalized electrons is a Lorentzian shape of width $\Gamma \simeq 1 / \pi \rho_{0}(0)$, for example, the local part of the Green function is given by

$$
\begin{aligned}
\frac{1}{N} \sum_{\mathbf{k}} G_{\sigma}\left(i \varepsilon_{n}, \mathbf{k}\right)= & \frac{1}{i \varepsilon_{n}+\mu-\epsilon_{a}-\tilde{\Sigma}_{\sigma}\left(i \varepsilon_{n}\right)+i \Gamma \operatorname{sgn}\left(\varepsilon_{n}\right)} \\
= & \frac{1-\langle n-\sigma\rangle}{i \varepsilon_{n}+\mu-\epsilon_{a}+i \Gamma \operatorname{sgn}\left(\varepsilon_{n}\right)} \\
& +\frac{\langle n-\sigma\rangle}{i \varepsilon_{n}+\mu-\epsilon_{a}-U+i \Gamma \operatorname{sgn}\left(\varepsilon_{n}\right)}
\end{aligned}
$$

with $\tilde{\Sigma}_{\sigma}\left(i \varepsilon_{n}\right)$ the selfenergy, $\mu$ the chemical potential, $\epsilon_{a}$ the band center, and $\left\langle n_{\sigma}\right\rangle$ the average number of electrons with spin $\sigma$ per site. When the Fermi-liquid relation 1011 is made use of, Gutzwiller's result gives the mass enhancement factor of

$$
\tilde{\phi}_{\gamma} \simeq 1 /(1-n)
$$

with $n=\left\langle n_{\uparrow}\right\rangle+\left\langle n_{\downarrow}\right\rangle$, so that the quasi-particle bandwidth is approximately given by $1 / \tilde{\phi}_{\gamma} \rho_{0}(0)$. Hubbard's result is relevant for single-particle excitations far from the chemical potential, while Gutzwiller's result is relevant for those in the vicinity of the chemical potential. A combined theory of Hubbard's and Gutzwiller's gives a three-peak structure of the density of states, Gutzwiller's band at the chemical potential between the lower and upper Hubbard bands. 
The selfenergy obtained by Hubbard does not depend on wave-numbers $\mathbf{k}$ and the mass enhancement factor obtained by Gutzwiller neither depends on k. The two theories are within the single-site approximation (SSA).

Treating the Hubbard or the periodic Anderson model in one of the best SSA's that include all the single-site terms is reduced to determining and solying selfconsistently a single-impurity Anderson model.12 The Anderson model is one of the simplest effective Hamiltonians for the Kondo problem The Kondo problem is almost completely understood, $13-16$ even the exact solution by the Bethe method was obtained. 17 Then, many useful results are available to clarify single-site properties in lattice systems. The most essential physics involved in the Kondo problem is the quenching of magnetic moments by local quantum spin fluctuation, so that the ground state is singlet and nonmagnetid 18 although it is close to a magnetic quantum critical point. No magnetic instability occurs within the SSA, and it can occur only when intersite or multi-site effects are properly considered. Multi-site effects can be perturbatively considered by starting from an unperturbed state constructed in the SSA. Because the quenching of magnetic moments by local quantum spin fluctuations is properly treated in such a perturbative theory, it must give a reliable magnetic instability condition. Such a perturbative theory is nothing but a theory of Kondo lattices. It is also nothing but a $1 / d$ expansion theory, with $d$ being spatial dimensionality. Any SSA that includes all the single-site terms is rigorous for Landau's Fermi-liquid states in infinite dimensions $(d \rightarrow+\infty) .19$ Multi-site terms are of higher order in $1 / d$ except for Weiss' mean fields, which are responsible for magnetic instability.

The spin susceptibility of magnets obeys the CurieWeiss law. Two mechanisms have been proposed for that of itinerant-electron magnets:20 the suppression of the spin susceptibility by the-made-mode coupling among intersite spin fluctyations 2123 and its enhancement by Weiss' mean fields.24.25 When the density of states is almost constant in the vicinity of the chemical potential, the suppression by the mode-mode coupling becomes larger withincreasing temperatures; it gives the Curie-Weiss law 2123 When the density of states has a sharp peak at the chemical potential, on the other hand, the suppression becomes larger with decreasing temperatures; the mode-mode coupling plays a totally negative role in the Curie-Weiss law in the flat-band model.25

Because the mode-mode coupling suppresses the spin susceptibility in any case, it cannot be a mechanism of magnetic instability. A scenario that the mechanism of the Curie-Weiss law is also responsible for magnetic instability itself seems to be more reasonable than the scenario that the mechanism of the Curie-Weiss law suppresses magnetic instability.

The mode-mode coupling is of higher order in $1 / d$ so that it vanishes in the large limit of $d$. There must be another mechanism, which is of leading order in $1 / d$. Only the possiblegne is the temperature dependence of Weiss' mean fields.20 The enhancement of the spin susceptibility by an exchange interaction arising from the virtual exchange of pair excitations of quasi-particles can give the Curie-Weiss law 24 Polarizations multiplied by the exchange interaction are Weiss' mean fields, and they are of leading order in $1 / d$. When the flat-band or band- edge conditions is satisfied, this exchange interaction is ferromagnetic and the Curie-Weiss law appears only in the homogeneous susceptibility. Miyai and the present author 25 examined the Curie-Weiss law of $\mathrm{Ni}$ by using Kanamori's model.

Field-induced ferromagnetism or metamagnetism is observed in many metals. In $\mathrm{CeRu}_{2} \mathrm{Si}_{2}$, for example, such a ferromagnetic state is stabilized at magnetic fields higher than $H_{M} \simeq 7.7 \mathrm{~T}$. One of the most crucialexperimental results is the single-parameter scaling;26.27 many quantities sensitively depend on pressures, and they scale with the Kondo temperature or energy, $k_{B} T_{K}$. The Kondo energy is approximately equal to a half of the bandwidth of quasiparticles, $k_{B} T_{K} \simeq 1 /\left[2 \tilde{\phi}_{\gamma} \rho_{0}(0)\right]$. This experiment gives a strong restriction for a ferromagnetic exchange interaction responsible for the metamagnetism in $\mathrm{CeRu}_{2} \mathrm{Si}_{2}$. Satoh and the present author showed that the exchange interaction responsible for the Curie-Weiss law is also responsible for the metamagnetism.28 In $4 f$ electron systems, the density of states is of a camelback structure or of a two-peak structure because of the hybridization between dispersive conduction bands and almost dispersionless $f$ bands. In the absence of magnetic fields, the chemical potential is between the two peaks; either of the flat-band and band-edge conditions is not satisfied, so that the exchange interaction is antiferromagnetic. In the presence of fields as high as $H_{M}$, the chemical potential is at one of the peaks of the camelback because of the Zeeman shift of up and down spin bands; both of the flat-band and band-edge conditions are satisfied, so that the exchange interaction becomes ferromagnetic around $H_{M}$. Because this exchange interaction has a novel property that its strength is proportional to the bandwidth of quasiparticles $1 / \tilde{\phi}_{\gamma} \rho(0)$, it is easy to explain the observed single-parameter scaling. Call this one simply a novel exchange interaction in this paper.

The previous paper 25.28 imply that when either or both of the flat-band and band-edge conditions are satisfied ferromagnetic instability is caused by the novel exchange interaction even in the absence of fields. Howeyer there is a crucial drawback in the previous papers.25. 28 An additional phenomenological ferromagnetic exchange interaction, which is independent of temperatures and magnetic fields, was assumed to obtain a quantitative agreement between experiment and theory. The authors of these papers speculated that such a ferromagnetic exchange interaction must exist in multi-band models.

The purpose of this paper is to study ferromagnetism in multi-band models from a theoretical viewpoint of the competition between magnetic instability caused by intersite exchange interactions and the quenching of magnetic moments by the Kondo effect. This paper is organized as follows: In Sec. II, an unperturbed state in the $1 / d$ expansion is constructed in the SSA. It is shown in Sec. III that when the Hund coupling is strong enough the superexchange interaction, which arises from the virtual exchange of pair excitations between the lower and upper Hubbard bands, becomes ferromagnetic. It is shown in Sec. IV that when the flat-band or band-edge condition is satisfied a ferromagnetic state can be stabilized. Discussion is given in Sec. V, and summary is given in Sec. VI. In Appendix A, a single-impurity Anderson model is studied by the $1 /(2 l+1)$ expansion method, with $2 l+1$ being orbital degeneracy. 


\section{PRELIMINARIES}

\section{A. Multi-band model with the Hund coupling}

Consider a $(2 l+1)$-band model, with $l$ an integer or a half integer:2s

$$
\mathcal{H}=\mathcal{H}_{0}+\mathcal{H}_{I}
$$

The first term is a one-body term given by

$$
\mathcal{H}_{0}=\sum_{m m^{\prime}} \sum_{\mathbf{k} \sigma}\left[E_{m m^{\prime}}(\mathbf{k})-\mu \delta_{m m^{\prime}}\right] a_{m \mathbf{k} \sigma}^{\dagger} a_{m^{\prime} \mathbf{k} \sigma},
$$

with $a_{m \mathbf{k} \sigma}^{\dagger}$ a creation operator of carriers, electrons or holes. Bands or orbitals are denoted by $m=-l,-(l-1)$, $\cdots$, and $l$, and up and down spins by $\sigma= \pm 1$. It is assumed that all the bands are degenerate at the $\Gamma$ point due to lattice symmetry, so that both $E_{m m}(0)$ and

$$
\epsilon_{a}=\frac{1}{N} \sum_{\mathbf{k}} E_{m m}(\mathbf{k})
$$

do not depend on $m$, and $E_{m m^{\prime}}(0)=0$ and $(1 / \sqrt{N}) \sum_{\mathbf{k}}$ $E_{m m^{\prime}}(\mathbf{k}) X_{i n v}(\mathbf{k})=0$ for $m \neq m^{\prime}$ and any function $X_{\text {inv }}(\mathbf{k})$ invariant under any symmetry transformation. In this paper, $E_{m m^{\prime}}(\mathbf{k})=E_{m^{\prime} m}(\mathbf{k})$ being real and

$$
E_{m m^{\prime}}(\mathbf{k})= \begin{cases}E_{l l}(\mathbf{k}), & m=m^{\prime} \\ E_{l-l}(\mathbf{k}), & m \neq m^{\prime}\end{cases}
$$

are also assumed. 30 This assumption makes arguments in this paper quite simple, as is shown later. The dispersion relations of $2 l+1$ bands are given by

$$
E_{m}^{*}(\mathbf{k})=\left\{\begin{array}{l}
E_{l l}(\mathbf{k})+2 l E_{l-l}(\mathbf{k}), m=l \\
E_{l l}(\mathbf{k})-E_{l-l}(\mathbf{k}),-l \leq m \leq(l-1)
\end{array} .\right.
$$

The flat-band condition is satisfied when the $l$ th band is almost dispersionless in such a way that

$$
E_{l}^{*}(\mathbf{k})=E_{l l}(\mathbf{k})+2 l E_{l-l}(\mathbf{k}) \simeq E_{l l}(0)
$$

for any $\mathbf{k}$. In this case, the dispersion relations of other $2 l$ bands $(m \neq l)$ are given by

$$
E_{m}^{*}(\mathbf{k}) \simeq E_{l l}(\mathbf{k})+\frac{1}{2 l}\left[E_{l l}(\mathbf{k})-E_{l l}(0)\right]
$$

The second term in Eq. (2.1) is the on-site Coulomb interaction:

$$
\begin{aligned}
\mathcal{H}_{I}= & \frac{1}{2} \sum_{m m^{\prime}} \sum_{i \sigma \sigma^{\prime}} U_{m m^{\prime}} a_{m i \sigma}^{\dagger} a_{m^{\prime} i \sigma^{\prime}}^{\dagger} a_{m^{\prime} i \sigma^{\prime}} a_{m i \sigma} \\
& +\frac{1}{2} J \sum_{m \neq m^{\prime}} \sum_{i \sigma \sigma^{\prime}} a_{m i \sigma}^{\dagger} a_{m^{\prime} i \sigma^{\prime}}^{\dagger} a_{m i \sigma^{\prime}} a_{m^{\prime} i \sigma} \\
= & \frac{1}{2} U \sum_{m i \sigma} n_{m i \sigma} n_{m i-\sigma} \\
& +\frac{1}{2}\left(U^{\prime}-\frac{1}{2} J\right) \sum_{m \neq m^{\prime}} \sum_{i \sigma \sigma^{\prime}} n_{m i \sigma} n_{m^{\prime} i \sigma^{\prime}} \\
& -J \sum_{m \neq m^{\prime}} \sum_{i X} \sum_{\sigma_{1} \sigma_{2}} \sum_{\sigma_{3} \sigma_{4}}\left(s_{X}^{\sigma_{1} \sigma_{2}} s_{X}^{\sigma_{3} \sigma_{4}}\right) \\
& \times a_{m i \sigma_{1}}^{\dagger} a_{m i \sigma_{2}} a_{m^{\prime} i \sigma_{3}}^{\dagger} a_{m^{\prime} i \sigma_{4}},
\end{aligned}
$$

with

$$
a_{m i \sigma}^{\dagger}=\frac{1}{\sqrt{N}} \sum_{\mathbf{k}} e^{-i\left(\mathbf{k} \cdot \mathbf{R}_{i}\right)} a_{m \mathbf{k} \sigma}^{\dagger}
$$

$a_{m i \sigma}$ its Hermite conjugate, and $n_{m i \sigma}=a_{m i \sigma}^{\dagger} a_{m i \sigma}$. Because of the degeneracy of $2 l+1$ bands at the $\Gamma$ point,

$$
U_{m m^{\prime}}= \begin{cases}U, & m=m^{\prime} \\ U^{\prime}, & m \neq m^{\prime}\end{cases}
$$

is also assumed. In general, the Hund coupling is ferromagnetic $(J>0)$. In Eq. (2.8b), $s_{X}^{\sigma \sigma^{\prime}}$ is the $\left(\sigma \sigma^{\prime}\right)$ th element of the $S=\frac{1}{2}$ spin matrix, $s_{x}, s_{y}$, or $s_{z}$. In this paper, $s_{z}$ is diagonalized so that $s_{z}^{\sigma \sigma^{\prime}}=\frac{1}{2} \sigma \delta_{\sigma \sigma^{\prime}}$.

\section{B. Mapping to the Anderson model}

The single-particle Green function for $\mathcal{H}$ is given by

$$
\mathcal{G}_{\sigma}^{-1}\left(i \varepsilon_{n}, \mathbf{k}\right)=\left(i \varepsilon_{n}+\mu\right) \mathcal{I}-\mathcal{E}(\mathbf{k})-\mathcal{S}_{\sigma}\left(i \varepsilon_{n}, \mathbf{k}\right)
$$

with $\mathcal{I}$ being the unit matrix, $[\mathcal{E}(\mathbf{k})]_{m m^{\prime}}=E_{m m^{\prime}}(\mathbf{k})$, and $\mathcal{S}_{\sigma}\left(i \varepsilon_{n}, \mathbf{k}\right)$ the selfenergy matrix. The selfenergy is divided into single-site and multisite terms:

$$
\mathcal{S}_{\sigma}\left(i \varepsilon_{n}, \mathbf{k}\right)=\tilde{\Sigma}_{\sigma}\left(i \varepsilon_{n}\right) \mathcal{I}+\Delta \mathcal{S}_{\sigma}\left(i \varepsilon_{n}, \mathbf{k}\right)
$$

with $\tilde{\Sigma}_{\sigma}\left(i \varepsilon_{n}\right)$ the single-site selfenergy and $\Delta \mathcal{S}_{\sigma}\left(i \varepsilon_{n}, \mathbf{k}\right)$ the multisite one. In our model, the selfenergy for $\mathbf{k}=0$ is proportional to the unit matrix because of the assumed symmetry; $\left[\Delta \mathcal{S}_{\sigma}\left(i \varepsilon_{n}, 0\right)\right]_{m m}$ does not depend on $m$ and $\left[\Delta \mathcal{S}_{\sigma}\left(i \varepsilon_{n}, 0\right)\right]_{m m^{\prime}}=0$ for $m \neq m^{\prime}$.

Calculating the single-site selfenergy $\tilde{\Sigma}_{\sigma}\left(i \varepsilon_{n}\right)$ is reduced to solving a single-impurity Anderson model:

$$
\begin{aligned}
\mathcal{H}_{A}= & \sum_{m \sigma}\left[\epsilon_{a}-\mu\right] n_{m \sigma}+\sum_{\mathbf{k} \sigma}\left[e_{c}(\mathbf{k})-\mu\right] c_{\mathbf{k} \sigma}^{\dagger} c_{\mathbf{k} \sigma} \\
& +\frac{1}{\sqrt{N}} \sum_{m \mathbf{k} \sigma}\left[v_{m \mathbf{k}} a_{m \sigma}^{\dagger} c_{\mathbf{k} \sigma}+v_{m \mathbf{k}}^{*} c_{\mathbf{k} \sigma}^{\dagger} a_{m \sigma}\right] \\
& +\frac{1}{2} \sum_{m m^{\prime}} \sum_{\sigma \sigma^{\prime}} U_{m m^{\prime}} a_{m \sigma}^{\dagger} a_{m^{\prime} \sigma^{\prime}}^{\dagger} a_{m^{\prime} \sigma^{\prime}} a_{m \sigma} \\
& +\frac{1}{2} J \sum_{m \neq m^{\prime}} \sum_{\sigma \sigma^{\prime}} a_{m \sigma^{\prime}}^{\dagger} a_{m^{\prime} \sigma^{\prime}}^{\dagger} a_{m \sigma^{\prime}} a_{m^{\prime} \sigma},
\end{aligned}
$$

with $n_{m \sigma}=a_{m \sigma}^{\dagger} a_{m \sigma}$. Here, $\epsilon_{a}, \mu, U_{m m^{\prime}}$, and $J$ are the same as those for the lattice system; $e_{c}(\mathbf{k})$ and $v_{m \mathbf{k}}$ should be determined so as to satisfy a mapping condition discussed below. The Green function for $\mathcal{H}_{A}$ is given by

$$
\tilde{\mathcal{G}}_{\sigma}^{-1}\left(i \varepsilon_{n}\right)=\left[i \varepsilon_{n}+\mu-\tilde{\Sigma}_{\sigma}\left(i \varepsilon_{n}\right)-\tilde{L}_{\sigma}\left(i \varepsilon_{n}\right)\right] \mathcal{I},
$$

with $\tilde{L}_{\sigma}\left(i \varepsilon_{n}\right)=(1 / N) \sum_{\mathbf{k}}\left|v_{m \mathbf{k}}\right|^{2} /\left[i \varepsilon_{n}-e_{c}(\mathbf{k})\right]$. Because of the assumed symmetry, $\tilde{\mathcal{G}}_{\sigma}^{-1}\left(i \varepsilon_{n}\right)$ is diagonal and $\tilde{L}_{\sigma}\left(i \varepsilon_{n}\right)$ does not depend on $m$. Because the symmetrical property of $\left[\Delta \mathcal{S}_{\sigma}\left(i \varepsilon_{n}, \mathbf{k}\right)\right]_{m m^{\prime}}$ is the same as that of $E_{m m^{\prime}}(\mathbf{k})$, the local Green function defined by

$$
\mathcal{R}_{\sigma}\left(i \varepsilon_{n}\right)=\frac{1}{N} \sum_{\mathbf{k}} \mathcal{G}_{\sigma}\left(i \varepsilon_{n}, \mathbf{k}\right)
$$


is also diagonal. When

$$
\tilde{\mathcal{G}}_{\sigma}\left(i \varepsilon_{n}\right)=\mathcal{R}_{\sigma}\left(i \varepsilon_{n}\right)
$$

is satisfied any single-site quantity of the lattice system is the same as its corresponding quantity of the singleimpurity Anderson model. The quantities, $\epsilon_{c}(\mathbf{k})$ and $v_{m \mathbf{k}}$, of the Anderson model should be selfconsistently determined to satisfy Eq. 2.16); Eq. (2.16) is nothing but the mapping condition to the Anderson model.

\section{EXCHANGE INTERACTIONS}

\section{A. Kondo lattices}

Consider the mapped Anderson model (2.13), and introduce fictitious and infinitesimally small external fields such as $\mathcal{H}_{\text {ext }}=-\sum_{m \sigma} \Delta E_{m \sigma} n_{m \sigma}$, with

$$
\Delta E_{m \sigma}=\Delta \mu+\mu_{B}\left[\frac{1}{2} \sigma g_{s} H_{s}+m g_{o} H_{o}+\frac{1}{2}(\sigma m) g_{s o} H_{s o}\right] \text {. }
$$

Here, $m$ is the Bohr magneton; $g_{s}, g_{o}$ and $g_{s o}$ are $g$ factors 31 The single-site selfenergy at $T=0 \mathrm{~K}$ is expanded in such a way that

$$
\begin{aligned}
\tilde{\Sigma}_{m \sigma}\left(i \varepsilon_{n}\right)= & \tilde{\Sigma}(0)+\left(1-\tilde{\phi}_{\gamma}\right) i \varepsilon_{n}+\left(1-\tilde{\phi}_{c}\right) \Delta \mu \\
& +\left(1-\tilde{\phi}_{s}\right) \frac{1}{2} \sigma g_{s} \mu_{B} H_{s}+\left(1-\tilde{\phi}_{l}\right) m g_{o} \mu_{B} H_{o} \\
& +\left(1-\tilde{\phi}_{s o}\right) \frac{1}{2}(\sigma m) g_{s o} \mu_{B} H_{s o}+\cdots
\end{aligned}
$$

for $\left|\varepsilon_{n}\right| \ll k_{B} T_{K}$, with $T_{K}$ the Kondo temperature discussed in Introduction and defined below.

When polarizations of conduction electrons are ignored, it follows according to Yoshimori's32 that

$$
\tilde{\phi}_{\gamma}=\frac{\tilde{\phi}_{c}}{2(2 l+1)}+\frac{3 \tilde{\phi}_{s}}{2(2 l+3)}+\frac{4 l(l+1) \tilde{\phi}_{o}}{(2 l+1)(2 l+3)},
$$

and static susceptibilities at $T=0 \mathrm{~K}$ of the Anderson model are simply given by

$$
\begin{gathered}
\tilde{\chi}_{c}(0) \equiv \frac{\partial}{\partial \Delta \mu} \sum_{m \sigma}\left\langle n_{m \sigma}\right\rangle=\tilde{\chi}_{c}^{*}(0) \\
\tilde{\chi}_{s}(0) \equiv \frac{\partial}{\partial H_{s}} \sum_{m \sigma} \frac{1}{2} \sigma g_{s} \mu_{B}\left\langle n_{m \sigma}\right\rangle=\frac{1}{4} g_{s}^{2} \mu_{B}^{2} \tilde{\chi}_{s}^{*}(0), \\
\tilde{\chi}_{o}(0) \equiv \frac{\partial}{\partial H_{o}} \sum_{m \sigma} m g_{o} \mu_{B}\left\langle n_{m \sigma}\right\rangle \\
=\frac{1}{3} l(l+1) g_{o}^{2} \mu_{B}^{2} \tilde{\chi}_{o}^{*}(0)
\end{gathered}
$$

and

$$
\begin{aligned}
\tilde{\chi}_{s o}(0) & \equiv \frac{\partial}{\partial H_{s o}} \sum_{m \sigma} \frac{1}{2}(m \sigma) g_{s o} \mu_{B}\left\langle n_{m \sigma}\right\rangle \\
& =\frac{1}{4} \cdot \frac{1}{3} l(l+1) g_{s o}^{2} \mu_{B}^{2} \tilde{\chi}_{s o}^{*}(0),
\end{aligned}
$$

with

$$
\tilde{\chi}_{\alpha}^{*}(0)=2(2 l+1) \tilde{\phi}_{\alpha} \rho(0)
$$

for charge $(\alpha=c)$, spin $(\alpha=s)$, band or orbital $(\alpha=o)$, and spin-orbital combined $(\alpha=s o)$ channels. Here, $\langle\cdots\rangle$ stands for the thermal average, and

$$
\rho(\varepsilon)=\frac{1}{2 l+1}\left(-\frac{1}{\pi}\right) \operatorname{Im}\left[\operatorname{Tr} \tilde{\mathcal{G}}_{\sigma}(\varepsilon+i 0)\right]
$$

is the density of states per spin and band.

So far, there is no restriction for $U, U^{\prime}$, or $J$. When $U=U^{\prime}$ and $J=0, \tilde{\phi}_{s}=\tilde{\phi}_{o}=\tilde{\phi}_{s o}$. It is reasonable to assume that $U \gg J, U^{\prime} \gg J$ and $U \gtrsim U^{\prime}$ so that $\tilde{\phi}_{s} \simeq \tilde{\phi}_{o} \simeq \tilde{\phi}_{s o}$. Only a single energy scale, the Kondo temperature, appears in low-energy phenomena of the single-site problem. In this paper, it is defined by

$$
k_{B} T_{K}=\frac{2 l+1}{\tilde{\chi}_{s}^{*}(0)}=2 \tilde{\phi}_{s} \rho(0) .
$$

The specific heat at low temperatures, $T \ll T_{K}$, is given by $\tilde{C}=\tilde{\gamma} T+\cdots$, with

$$
\tilde{\gamma}=\frac{2(2 l+1)}{3} \tilde{\phi}_{\gamma} \pi^{2} k_{B}^{2} \rho(0)
$$

The Kondo temperature, $k_{B} T_{K}$, is as large as the effective Fermi energy of quasiparticles in the lattice system.

In this paper, the Kondo temperature is treated as a phenomenological parameter instead of determining and solving the Anderson model selfconsistently.

Define a two-point polarization function of the lattice system, which is a $2(2 l+1) \times 2(2 l+1)$ matrix, by

$$
\begin{aligned}
{\left[\Pi\left(i \omega_{l}, \mathbf{q}\right)\right]_{m \sigma, m^{\prime} \sigma^{\prime}}=} & \frac{1}{N} \sum_{i j} \int_{0}^{1 / k_{B} T} d \tau e^{i \omega_{l} \tau+i \mathbf{q} \cdot\left(\mathbf{R}_{i}-\mathbf{R}_{j}\right)} \\
& \times\left\langle n_{m i \sigma}(\tau) n_{m^{\prime} j \sigma^{\prime}}(0)\right\rangle
\end{aligned}
$$

When its irreducible part is denoted by $\boldsymbol{\pi}\left(i \omega_{l}, \mathbf{q}\right)$,

$$
\boldsymbol{\Pi}\left(i \omega_{l}, \mathbf{q}\right)=\boldsymbol{\pi}\left(i \omega_{l}, \mathbf{q}\right)\left[1+\mathcal{U} \boldsymbol{\pi}\left(i \omega_{l}, \mathbf{q}\right)\right]^{-1} .
$$

According to Eq. (2.8b), $\mathcal{U}$ is defined by

$$
[\mathcal{U}]_{m \sigma, m^{\prime} \sigma^{\prime}}=\left\{\begin{array}{cl}
0, & m=m^{\prime} \text { and } \sigma=\sigma^{\prime} \\
U^{\prime}-J, & m \neq m^{\prime} \text { and } \sigma=\sigma^{\prime} \\
U, & m=m^{\prime} \text { and } \sigma \neq \sigma^{\prime} \\
U^{\prime}, & m \neq m^{\prime} \text { and } \sigma \neq \sigma^{\prime}
\end{array} .\right.
$$

The irreducible part is divided into a single-site term $\tilde{\boldsymbol{\pi}}\left(i \omega_{l}\right)$ and a multisite term $\Delta \boldsymbol{\pi}\left(i \omega_{l}, \mathbf{q}\right)$ so that

$$
\boldsymbol{\pi}\left(i \omega_{l}, \mathbf{q}\right)=\tilde{\boldsymbol{\pi}}\left(i \omega_{l}\right)+\Delta \boldsymbol{\pi}\left(i \omega_{l}, \mathbf{q}\right)
$$

The reducible polarization function of the mapped Anderson model is given by

$$
\tilde{\boldsymbol{\Pi}}\left(i \omega_{l}\right)=\tilde{\boldsymbol{\pi}}\left(i \omega_{l}\right)\left[1+\mathcal{U} \tilde{\boldsymbol{\pi}}\left(i \omega_{l}\right)\right]^{-1}
$$

Define the following $2(2 l+1) \times 2(2 l+1)$ matrix: 


$$
K_{m \sigma, m^{\prime} \sigma^{\prime}}^{(\alpha)}=\left\{\begin{array}{cc}
\frac{1}{2(2 l+1)}, & \alpha=c \\
\frac{1}{2(2 l+1)} \sigma \sigma^{\prime}, & \alpha=s \\
\frac{1}{2(2 l+1)} \frac{3 m m^{\prime}}{l(l+1)}, & \alpha=o \\
\frac{1}{2(2 l+1)} \frac{3 m m^{\prime} \sigma \sigma^{\prime}}{l(l+1)}, & \alpha=s o
\end{array} .\right.
$$

Because our system has the particular symmetry such as Eqs. (2.4), (2.10) and (3.14), susceptibilities of the four channels are simply given by

$$
\begin{aligned}
\chi_{\alpha}^{*}\left(i \omega_{l}, \mathbf{q}\right) & \equiv 2(2 l+1) \sum_{m \sigma} \sum_{m^{\prime} \sigma^{\prime}} K_{m \sigma, m^{\prime} \sigma^{\prime}}^{(\alpha)}\left[\Pi\left(i \omega_{l}, \mathbf{q}\right)\right]_{m \sigma, m^{\prime} \sigma^{\prime}} \\
& =2(2 l+1) \frac{\pi_{\alpha}\left(i \omega_{l}, \mathbf{q}\right)}{1-U_{\alpha} \pi_{\alpha}\left(i \omega_{l}, \mathbf{q}\right)}
\end{aligned}
$$

and

$$
\begin{aligned}
\tilde{\chi}_{\alpha}^{*}\left(i \omega_{l}\right) & \equiv 2(2 l+1) \sum_{m \sigma} \sum_{m^{\prime} \sigma^{\prime}} K_{m \sigma, m^{\prime} \sigma^{\prime}}^{(\alpha)}\left[\tilde{\Pi}\left(i \omega_{l}\right)\right]_{m \sigma, m^{\prime} \sigma^{\prime}} \\
& =2(2 l+1) \frac{\tilde{\pi}_{\alpha}\left(i \omega_{l}\right)}{1-U_{\alpha} \tilde{\pi}_{\alpha}\left(i \omega_{l}\right)}
\end{aligned}
$$

with

$$
\begin{gathered}
U_{\alpha}=-\sum_{m \sigma} \sum_{m^{\prime} \sigma^{\prime}} K_{m \sigma, m^{\prime} \sigma^{\prime}}^{(\alpha)}[\mathcal{U}]_{m \sigma, m^{\prime} \sigma^{\prime}} \\
=\left\{\begin{array}{cl}
-U-2 l\left(2 U^{\prime}-J\right), & \alpha=c \\
U+2 l J, & \alpha=s \\
-U+2 U^{\prime}-J, & \alpha=o \\
U-J, & \alpha=s o
\end{array}\right.
\end{gathered}
$$

Here, $\pi_{\alpha}\left(i \omega_{l}, \mathbf{q}\right)$ and $\tilde{\pi}_{\alpha}\left(i \omega_{l}\right)$ are irreducible polarization functions defined by

$$
\begin{gathered}
\tilde{\pi}_{\alpha}\left(i \omega_{l}\right)=\sum_{m \sigma} \sum_{m^{\prime} \sigma^{\prime}} K_{m \sigma, m^{\prime} \sigma^{\prime}}^{(\alpha)}\left[\tilde{\boldsymbol{\pi}}\left(i \omega_{l}\right)\right]_{m \sigma, m^{\prime} \sigma^{\prime}} \\
\Delta \pi_{\alpha}\left(i \omega_{l}, \mathbf{q}\right)=\sum_{m \sigma} \sum_{m^{\prime} \sigma^{\prime}} K_{m \sigma, m^{\prime} \sigma^{\prime}}^{(\alpha)}\left[\Delta \boldsymbol{\pi}\left(i \omega_{l}, \mathbf{q}\right)\right]_{m \sigma, m^{\prime} \sigma^{\prime}},
\end{gathered}
$$

and

$$
\pi_{\alpha}\left(i \omega_{l}, \mathbf{q}\right)=\tilde{\pi}_{\alpha}\left(i \omega_{l}\right)+\Delta \pi_{\alpha}\left(i \omega_{l}, \mathbf{q}\right) .
$$

Note that $\tilde{\chi}_{\alpha}^{*}\left(i \omega_{l}\right)$ is nothing but the susceptibility of the mapped Anderson model.

It follows from Eqs. (3.18) and (3.19) that

$$
\chi_{\alpha}^{*}\left(i \omega_{l}, \mathbf{q}\right)=\frac{\tilde{\chi}_{\alpha}^{*}\left(i \omega_{l}\right)}{1-\frac{1}{4} I_{\alpha}\left(i \omega_{l}, \mathbf{q}\right) \tilde{\chi}_{\alpha}^{*}\left(i \omega_{l}\right)},
$$

with

$$
I_{\alpha}\left(i \omega_{l}, \mathbf{q}\right)=\frac{2}{2 l+1} \cdot \frac{\Delta \pi_{\alpha}\left(i \omega_{l}, \mathbf{q}\right)}{\tilde{\pi}_{\alpha}\left(i \omega_{l}\right) \pi_{\alpha}\left(i \omega_{l}, \mathbf{q}\right)} .
$$

In this paper, the strong coupling regime is defined by

$$
U_{s} \gg k_{B} T_{K}
$$

instead of Eq. (1.1). When either of the flat-band and band-edge conditions is satisfied, Eq. (3.26) is satisfied. Eq. (3.26) leads to a set of $U_{\alpha} \tilde{\chi}_{\alpha}^{*}(0) \gg 1$ and $U_{\alpha} \chi_{\alpha}^{*}(0, \mathbf{q}) \gg 1$ except for $\alpha=c$. Then, as long as $\left|\omega_{l}\right| \lesssim k_{B} T_{K}$,

$$
U_{\alpha} \tilde{\pi}_{\alpha}\left(i \omega_{l}\right)=1+O\left(1 / U_{\alpha} \tilde{\chi}_{\alpha}^{*}(0)\right)
$$

and $U_{\alpha} \pi_{\alpha}\left(i \omega_{l}, \mathbf{q}\right)=1+O\left(1 / U_{\alpha} \chi_{\alpha}^{*}(0, \mathbf{q})\right)$ so that

$$
I_{\alpha}\left(i \omega_{l}, \mathbf{q}\right)=\frac{2}{2 l+1} U_{\alpha}^{2} \Delta \pi_{\alpha}\left(i \omega_{l}, \mathbf{q}\right)
$$

The susceptibility given by Eq. (3.24) is consistent with a physical picture for Kondo lattices, where local fluctuations at different sites interact with each other by intersite exchange interactions. Then, we call $I_{\alpha}\left(i \omega_{l}, \mathbf{q}\right)$ given by Eq. (3.25) or (3.28) an intersite exchange interaction for the spin, orbital, or combined channel. A perturbative treatment of $I_{\alpha}\left(i \omega_{l}, \mathbf{q}\right)$ is nothing but the $1 / d$ expansion; $I_{\alpha}\left(i \omega_{l}, \mathbf{q}\right)$ is of higher order in $1 / d$ for almost all q's, and it is leading order in $1 / d$ for particular $\mathbf{q}$ 's such as $\mathbf{q}=0$, nesting wave vectors, and so on.
FIG. 1. Examples of two-line diagrams for $\Delta \pi_{\alpha}\left(i \omega_{l}, \mathbf{q}\right)$. Diagram $(a)$, which is in the site representation, gives the superexchange interaction between the $i$ th and $j$ th sites. Diagram $(b)$, which is in the wave-number representation, gives the novel exchange interaction, which arises from the virtual exchange of pair excitations of quasi-particles with wave numbers $\mathbf{k}+\mathbf{q}$ and $\mathbf{k}$. Two-line diagrams higher order in intersite effects such as $(c)$ can be still of leading order in $1 / d$, but they are ignored in this paper. A solid line stands for the single-particle Green function, $\tilde{G}_{m \sigma}\left(i \varepsilon_{n}\right)$ given by Eq. (3.31) in (a) or the intersite part of $\mathcal{G}_{\sigma}\left(i \varepsilon_{n}, \mathbf{k}+\mathbf{q}\right)$ or $\mathcal{G}_{\sigma}\left(i \varepsilon_{n}, \mathbf{k}\right)$ given by Eqs. (3.49) and (3.50) in (b). A cross in $(a)$ stands for $t_{m i, m^{\prime} j}$ given by Eq. (3.38), a shaded triangle for the single-site irreducible three-point vertex function given by Eq. (3.34) in $(a)$ or Eq. (3.51) in $(b)$, and a shaded rectangle in $(c)$ for the single-site irreducible four-point vertex function.

In general, an exchange interaction arises from the virtual exchange of bosons or bosonic excitations. The exchange interaction given by Eq. (3.28) is divided into four terms in such a way that 


$$
\begin{aligned}
I_{\alpha}\left(i \omega_{l}, \mathbf{q}\right)=I_{\alpha}^{(s)} & \left(i \omega_{l}, \mathbf{q}\right)+I_{\alpha}^{(Q)}\left(i \omega_{l}, \mathbf{q}\right) \\
& +\Delta I_{\alpha}^{(s Q)}\left(i \omega_{l}, \mathbf{q}\right)-4 \Lambda_{\alpha}\left(i \omega_{l}, \mathbf{q}\right) .
\end{aligned}
$$

Only contributions from the so called two-line diagrams can be of leading order in $1 / d$. Three examples of two-line diagrams are shown in Fig. 11. The first term, $I_{\alpha}^{(s)}\left(i \omega_{l}, \mathbf{q}\right)$, corresponds to Fig. $\mathrm{I}(a)$, and is due to the virtual exchange of pair excitations of electrons or holes across the Mott-Hubbard gap, whose excitation energies are as large as $U$ or $U^{\prime} \pm J$ and much larger than $k_{B} T_{K}$. This is a generalized superexchange interaction. The second term, $I_{\alpha}^{(Q)}\left(i \omega_{l}, \mathbf{q}\right)$, corresponds to Fig. 1 $1(b)$, and is due to the virtual exchange of pair excitations of quasiparticles, whose excitation energies are $O\left(k_{B} T_{K}\right)$. This is the novel exchange interaction discussed in Introduction. The third term, $\Delta I_{\alpha}^{(s Q)}\left(i \omega_{l}, \mathbf{q}\right)$, is the contribution from two-line diagrams higher order in intersite effects such as Fig. 1 $1(c)$. The last term, $-4 \Lambda_{\alpha}\left(i \omega_{l}, \mathbf{q}\right)$, includes all the other contributions, which are definitely higher order in $1 / d$. Its lowest-order Feynman diagrams correspond to those of the mode-mode coupling terms considered in the so called selfconsistent renormalization (SCR) theory of spin fluctuations.22, 23

The superexchange interaction, $I_{\alpha}^{(s)}\left(i \omega_{l}, \mathbf{q}\right)$, is considered in Sec. IIIB, and the novel exchange interaction, $I_{\alpha}^{(Q)}\left(i \omega_{l}, \mathbf{q}\right)$, is considered in Sec. III . On the other hand, $\Lambda_{\alpha}\left(i \omega_{l}, \mathbf{q}\right)$ as well as $\Delta I_{\alpha}^{(s Q)}\left(i \omega_{l}, \mathbf{q}\right)$ are ignored in this paper. Because $\Lambda_{\alpha}\left(i \omega_{l}, \mathbf{q}\right)$ is of higher order in $1 / d$ for any $\mathbf{q}$, it can be ignored at least for large enough $d$.

\section{B. Superexchange interaction}

There is no restriction for carrier densities so far. In the following part of this paper, our study is restricted to low densities of carriers 34

$$
\left\langle n_{m \sigma}\right\rangle \ll 1, \quad n=\sum_{m \sigma}\left\langle n_{m \sigma}\right\rangle<1,
$$

so that multiply occupied configurations at a single site can be ignored in the ground state and low lying excited states. The single-particle Green function is approximately given by $\left[\tilde{\mathcal{G}}_{\sigma}\left(i \varepsilon_{n}\right)\right]_{m m^{\prime}}=\delta_{m m^{\prime}} \tilde{G}_{m \sigma}\left(i \varepsilon_{n}\right)$, with

$$
\begin{aligned}
\tilde{G}_{m \sigma}\left(i \varepsilon_{n}\right)= & \frac{\left\langle n_{m \sigma}\right\rangle+n_{e}}{i \varepsilon_{n}+\mu-\epsilon_{a}+i \Gamma \operatorname{sgn}\left(\varepsilon_{n}\right)} \\
& +\frac{\left\langle n_{m-\sigma}\right\rangle}{i \varepsilon_{n}+\mu-\epsilon_{a}-U+i \Gamma \operatorname{sgn}\left(\varepsilon_{n}\right)} \\
& +\sum_{m^{\prime} \neq m}\left[\frac{\left\langle n_{m^{\prime} \sigma}\right\rangle+\frac{1}{2}\left\langle n_{m^{\prime}-\sigma}\right\rangle}{i \varepsilon_{n}+\mu-\epsilon_{a}-U^{\prime}+J+i \Gamma \operatorname{sgn}\left(\varepsilon_{n}\right)}\right. \\
& \left.+\frac{\frac{1}{2}\left\langle n_{m^{\prime}-\sigma}\right\rangle}{i \varepsilon_{n}+\mu-\epsilon_{a}-U^{\prime}-J+i \Gamma \operatorname{sgn}\left(\varepsilon_{n}\right)}\right] \\
& +\cdots \cdots .
\end{aligned}
$$

Here, $\pi \Gamma \simeq 1 / \rho_{0}(0)$ and

$$
n_{e} \simeq 1-\sum_{m^{\prime} \sigma^{\prime}}\left\langle n_{m^{\prime} \sigma^{\prime}}\right\rangle
$$

is the density of empty sites. This is a straightforward extension of Eq. (1.2) or Hubbard's theory

According to the Ward identity, 35 the reducible singlesite three-point vertex function is given by

$$
\left[\tilde{\Lambda}\left(i \varepsilon_{n}, i \varepsilon_{n} ; 0\right)\right]_{m \sigma, m^{\prime} \sigma^{\prime}}=\frac{\partial \tilde{G}_{m \sigma}^{-1}\left(i \varepsilon_{n}\right)}{\partial \Delta E_{m^{\prime} \sigma^{\prime}}} .
$$

When only the dependence of $\left\langle n_{m \sigma}\right\rangle$ on $\Delta E_{m^{\prime} \sigma^{\prime}}$ is considered in Eq. (3.31), the irreducible single-site three-point vertex function is calculated in such a way that

$$
\begin{aligned}
\tilde{\lambda}_{\alpha}\left(i \varepsilon_{n}, i \varepsilon_{n} ; 0\right)= & \sum_{m \sigma} \sum_{m^{\prime} \sigma^{\prime}} K_{m \sigma, m^{\prime} \sigma^{\prime}}^{(\alpha)} \\
& \times\left[\tilde{\boldsymbol{\Lambda}}\left(i \varepsilon_{n}, i \varepsilon_{n} ; 0\right)[1-\mathcal{U} \tilde{\boldsymbol{\pi}}(0)]\right]_{m \sigma, m^{\prime} \sigma^{\prime}} \\
= & -\frac{\tilde{\pi}_{\alpha}(0)}{\tilde{G}_{m \sigma}^{2}\left(i \varepsilon_{n}\right)} \sum_{r=1}^{4} \frac{A_{\alpha ; r}}{i \varepsilon_{n}-z_{r}+i \Gamma \operatorname{sgn}\left(\varepsilon_{n}\right)},
\end{aligned}
$$

with

$$
\left(\begin{array}{c}
z_{1} \\
z_{2} \\
z_{3} \\
z_{4}
\end{array}\right)=\left(\begin{array}{c}
\epsilon_{a}-\mu \\
\epsilon_{a}+U-\mu \\
\epsilon_{a}+U^{\prime}-J-\mu \\
\epsilon_{a}+U^{\prime}+J-\mu
\end{array}\right)
$$

and

$$
\left(\begin{array}{ccc}
A_{s ; 1} & A_{o ; 1} & A_{s o: 1} \\
A_{s ; 2} & A_{o ; 2} & A_{s o: 2} \\
A_{s ; 3} & A_{o ; 3} & A_{s o: 3} \\
A_{s ; 4} & A_{o ; 4} & A_{s o: 4}
\end{array}\right)=\left(\begin{array}{rrr}
1 & 1 & 1 \\
-1 & 1 & -1 \\
l & -\frac{3}{2} & -\frac{1}{2} \\
-l & -\frac{1}{2} & \frac{1}{2}
\end{array}\right),
$$

for spin $(\alpha=s)$, orbital $(\alpha=o)$, and combined $(\alpha=s o)$ channels.

Consider an exchange interaction between the $i$ th and $j$ th sites, which is defined by

$$
I_{\alpha ; i j}^{(s)}\left(i \omega_{l}\right)=\frac{1}{N} \sum_{\mathbf{q}} I_{\alpha}^{(s)}\left(i \omega_{l}, \mathbf{q}\right) e^{-i \mathbf{q} \cdot\left(\mathbf{R}_{i}-\mathbf{R}_{j}\right)},
$$

with $\mathbf{R}_{i}$ being the position of the $i$ th unit cell. Transfer integrals between the $i$ th and $j$ th sites have also the following symmetry 36

$$
\begin{aligned}
t_{m i, m^{\prime} j} & =-\frac{1}{N} \sum_{\mathbf{k}} E_{m m^{\prime}}(\mathbf{k}) e^{i \mathbf{k} \cdot\left(\mathbf{R}_{i}-\mathbf{R}_{j}\right)} \\
& \equiv \begin{cases}t_{i j}, & m=m^{\prime} \\
t_{i j}^{\prime}, & m \neq m^{\prime}\end{cases}
\end{aligned}
$$

When the two-line diagram shown in Fig. 1 $1(a)$ is taken into account and Eq. (3.27) is made use of, the superexchange interaction is calculated in such a way that

$$
I_{\alpha ; i j}^{(s)}(0)=\frac{2}{2 l+1} \cdot \frac{2}{U} T_{i j}^{(\alpha)} Y_{\alpha ; i j},
$$

with

$$
\begin{aligned}
T_{i j}^{(\alpha)} & =\sum_{m m^{\prime} \sigma} K_{m \sigma, m^{\prime} \sigma}^{(\alpha)}\left|t_{m i, m^{\prime} j}\right|^{2} \\
& = \begin{cases}\left|t_{i j}\right|^{2}+2 l\left|t_{i j}^{\prime}\right|^{2}, & \alpha=s \\
\left|t_{i j}\right|^{2}-\left|t_{i j}^{\prime}\right|^{2}, & \alpha=o \text { and } s o\end{cases}
\end{aligned}
$$


and

$$
\begin{aligned}
Y_{\alpha ; i j} & =\frac{1}{2} U\left(-k_{B} T\right) \sum_{\varepsilon_{n}} U_{\alpha}^{2} \tilde{\lambda}_{\alpha}^{2}\left(i \varepsilon_{n}, i \varepsilon_{n} ; 0\right) \tilde{G}_{m \sigma}^{4}\left(i \varepsilon_{n}\right) \\
& =\frac{1}{2} U \sum_{r=1}^{4} \sum_{r^{\prime}=1}^{4} A_{\alpha ; r} A_{\alpha ; r^{\prime}} \Xi\left(z_{r}, z_{r^{\prime}} ; \Gamma\right) .
\end{aligned}
$$

Here, $\Xi\left(z, z^{\prime} ; \Gamma\right)$ is defined in such a way that

$$
\Xi\left(z, z^{\prime} ; \Gamma\right) \equiv \frac{1}{\pi\left(z-z^{\prime}\right)}\left[\operatorname{Tan}^{-1}\left(\frac{z}{\Gamma}\right)-\operatorname{Tan}^{-1}\left(\frac{z^{\prime}}{\Gamma}\right)\right]
$$

for $z \neq z^{\prime}$, and $\Xi(z, z)=\lim _{z^{\prime} \rightarrow z} \Xi\left(z, z^{\prime}\right)$. Note that $Y_{\alpha ; i j}=-1$ for $\Gamma / U \rightarrow+0$ and $J=0$. When the carrier density is so small that Eq. (3.30) is satisfied, the contribution from excitations within the lower Hubbard band $\left(r=r^{\prime}=1\right)$ is nothing but that from the virtual exchange of pair excitations of quasi-particles. Because it is considered in the next subsection, it should be subtracted in order togvoid double counting so that Eq. (3.41) is replaced by 37

$$
Y_{\alpha ; i j}=\frac{1}{2} U \sum_{r \neq r^{\prime}} A_{\alpha ; r} A_{\alpha ; r^{\prime}} \Xi\left(z_{r}, z_{r^{\prime}} ; \Gamma\right)
$$

In the following part, $I_{\alpha ; i j}^{(s)}\left(i \omega_{l}=0\right)$ is simply denoted by $I_{\alpha ; i j}^{(s)}$. Because its $\omega_{l}$ dependence is small, it can be used for $\left|\omega_{l}\right| \ll U$ and $U^{\prime}-J$.

In the small limit of $\Gamma / U \rightarrow+0$, it follows that

$$
\begin{gathered}
I_{s ; i j}^{(s)}=-\frac{4 T_{i j}^{(s)}}{2 l+1}\left[\frac{1}{U}-l\left(\frac{1}{U^{\prime}-J}-\frac{1}{U^{\prime}+J}\right)\right], \\
I_{o ; i j}^{(s)}=-\frac{4 T_{i j}^{(o)}}{2 l+1}\left[-\frac{1}{U}+\frac{1}{2}\left(\frac{3}{U^{\prime}-J}+\frac{1}{U^{\prime}+J}\right)\right],
\end{gathered}
$$

and

$$
I_{s o: i j}^{(s)}=-\frac{4 T_{i j}^{(s o)}}{2 l+1}\left[\frac{1}{U}+\frac{1}{2}\left(\frac{1}{U^{\prime}-J}-\frac{1}{U^{\prime}+J}\right)\right] .
$$

In deriving these results, $\epsilon_{a}-\mu \ll-k_{B} T, \epsilon_{a}+U-\mu \gg$ $k_{B} T$ and $\epsilon_{a}+U^{\prime}-J-\mu \gg k_{B} T$ are assumed. These results agree with those calculated for insulating phases by the conventional perturbative method. 33

Note that Eq. (3.44a) can be applied even to $l=0$. The superexchange interaction in spin channels can never be ferromagnetic in the single-band model $(l=0)$. It can be ferromagnetic in multi-band models $(l \geq 1 / 2)$, when $J$ is as large as

$$
J>\sqrt{l^{2} U^{2}+\left(U^{\prime}\right)^{2}}-l U .
$$

It is interesting that as long as $J>0$ the superexchange interaction is definitely ferromagnetic in the large limit of orbital multiplicity $(l \rightarrow+\infty)$.

Non-zero $\Gamma$ makes the superexchange interaction reduced. Fig. 2 shows $Y_{s ; i j}$ as a function of $J$ for various $\Gamma$ 's, and Fig. 3 shows $Y_{s ; i j}$ as a function of $\epsilon_{a}-\mu$ for the same $\Gamma$ 's. Positive and negative $Y_{s ; i j}$ 's mean that the superexchange interaction is ferromagnetic and antiferromagnetic, respectively. When $J$ and $l$ are large enough, the superexchange interaction is ferromagnetic even for non-zero $\Gamma$.

FIG. 2. $\quad Y_{s ; i j}$ defined by Eq. 3.43 as a function of the Hund coupling, $J$, for $l=1$ and $l=2$. Here, $\left(\epsilon_{a}-\mu\right) / U=-0.1$ and $U^{\prime} / U=3 / 4$ are assumed. A solid line shows $Y_{s ; i j}$ for $\pi \Gamma / U=0.3$, a broken line for $\pi \Gamma / U=0.2$, a dotted-broken line for $\pi \Gamma / U=0.1$, and a dotted line for $\pi \Gamma / U=0$.

FIG. 3. $Y_{s ; i j}$ defined by Eq. (3.43) as a function of the depth, $\epsilon_{a}-\mu$, for $l=1$ and $l=2$. Here, $J / U=0.3$ and $U^{\prime} / U=3 / 4$ are assumed. A solid line shows $Y_{s ; i j}$ for $\pi \Gamma / U=0.3$, a broken line for $\pi \Gamma / U=0.2$, a dotted-broken line for $\pi \Gamma / U=0.1$, and a dotted line for $\pi \Gamma / U=0$.

The signs of $I_{o ; i j}^{(s)}$ and $I_{s o: i j}^{(s)}$ depend on $\left|t_{i j}\right|^{2}-\left|t_{i j}^{\prime}\right|^{2}$. When $\left|t_{i j}\right| \simeq\left|t_{i j}^{\prime}\right|$, both of $I_{o: i j}^{(s)}$ and $I_{s o: i j}^{(s)}$ are small. Interesting is the case where either of $\left|t_{i j}\right|^{2}$ and $\left|t_{i j}^{\prime}\right|^{2}$ is much larger than the other, because it is possible that an 
instability of the orbital or combined channel occurs at low enough channels. In general, $\left|I_{o ; i j}^{(s)}\right|>\left|I_{s o ; i j}^{(s)}\right|$.

\section{Novel exchange interaction}

Selfenergy corrections from multi-site effects are of higher order in $1 / d$ except for those due to Weiss' mean fields, which are not present in Landau's normal Fermiliquid phases. When only the single-site selfenergy given by Eq. (3.2) is taken into account, the single-particle Green function is approximately given by

$$
\left[\mathcal{G}_{\sigma}^{-1}\left(i \varepsilon_{n}, \mathbf{k}\right)\right]_{m m^{\prime}}=\tilde{\phi}_{\gamma}\left[i \varepsilon_{n} \delta_{m m^{\prime}}-e_{m m^{\prime}}(\mathbf{k})\right],
$$

with

$$
e_{m m^{\prime}}(\mathbf{k})=\frac{1}{\tilde{\phi}_{\gamma}}\left[E_{m m^{\prime}}(\mathbf{k})+\left(\tilde{\Sigma}_{0}(0)-\mu\right) \delta_{m m^{\prime}}\right],
$$

for $\left|\varepsilon_{n}\right| \lesssim k_{B} T_{K}$. The dispersion relation of quasiparticles, $\xi_{m}(\mathbf{k})$, is determined from $\operatorname{det}\left[\mathcal{G}_{\sigma}^{-1}\left(\xi_{m}(\mathbf{k}), \mathbf{k}\right)\right]=0$, so that

$$
\xi_{m}(\mathbf{k})=\frac{1}{\tilde{\phi}_{\gamma}}\left[\tilde{\Sigma}_{0}(0)+E_{m}^{*}(\mathbf{k})-\mu\right]
$$

with $E_{m}^{*}(\mathbf{k})$ given by Eq. (2.5). Note that $\xi_{m}(\mathbf{k})=\xi_{-l}(\mathbf{k})$ for $m$ such as $-l \leq m \leq(l-1)$ and only $\xi_{l}(\mathbf{k})$ can be different from them. The $\left(\mathrm{mm}^{\prime}\right)$ th component of the Green function, is calculated in such a way that

$$
\left[\mathcal{G}_{\sigma}\left(i \varepsilon_{n}, \mathbf{k}\right)\right]_{m m}=\frac{1}{(2 l+1) \tilde{\phi}_{\gamma}}\left[\frac{2 l}{i \varepsilon_{n}-\xi_{-l}(\mathbf{k})}+\frac{1}{i \varepsilon_{n}-\xi_{l}(\mathbf{k})}\right]
$$

for intraband components, and

$$
\left[\mathcal{G}_{\sigma}\left(i \varepsilon_{n}, \mathbf{k}\right)\right]_{m m^{\prime}}=\frac{(-1)^{2 l+1}}{(2 l+1) \tilde{\phi}_{\gamma}}\left[\frac{1}{i \varepsilon_{n}-\xi_{-l}(\mathbf{k})}-\frac{1}{i \varepsilon_{n}-\xi_{l}(\mathbf{k})}\right]
$$

for interband components $\left(m \neq m^{\prime}\right)$.

According to the Ward identity 35

$$
\begin{aligned}
\tilde{\lambda}_{\alpha}(0,0 ; 0) & =\left[1-U_{\alpha} \tilde{\pi}_{\alpha}(0)\right] \tilde{\phi}_{\alpha} \\
& =\frac{2(2 l+1) \tilde{\phi}_{\alpha}}{U_{\alpha} \tilde{\chi}_{\alpha}^{*}(0)}\left[1+O\left(\frac{1}{U_{\alpha} \tilde{\chi}_{\alpha}^{*}(0)}\right)\right] .
\end{aligned}
$$

When this is approximately used for non-zero energies such as $\left|\varepsilon_{n}\right| \lesssim k_{B} T_{k}$ and $\left|\omega_{l}\right| \lesssim k_{B} T_{k}$ and the two-line diagram shown in Fig. 1 $1(b)$ is taken into account, the exchange interaction due to the virtual exchange of pair excitations of quasiparticles is calculated so that

$$
\begin{aligned}
I_{\alpha}^{(Q)}\left(i \omega_{l}, \mathbf{q}\right) & =\left[U_{\alpha} \tilde{\lambda}_{\alpha}(0,0 ; 0)\right]^{2} \frac{1}{\tilde{\phi}_{\gamma}^{2}}\left[P_{\alpha}\left(i \omega_{l}, \mathbf{q}\right)-\tilde{P}_{\alpha}\left(i \omega_{l}\right)\right] \\
& =\left(\frac{\tilde{\phi}_{\alpha}}{\tilde{\phi}_{\gamma}}\right)^{2} \frac{8(2 l+1)}{\left[\tilde{\chi}_{\alpha}^{*}(0)\right]^{2}}\left[P_{\alpha}\left(i \omega_{l}, \mathbf{q}\right)-\tilde{P}_{\alpha}\left(i \omega_{l}\right)\right] .
\end{aligned}
$$

$$
\begin{aligned}
P_{\alpha}\left(i \omega_{l}, \mathbf{q}\right)= & -k_{B} T \sum_{\varepsilon_{n}} \frac{1}{N} \sum_{\mathbf{k}} \sum_{m m^{\prime} \sigma} K_{m \sigma, m^{\prime} \sigma}^{(\alpha)} \tilde{\phi}_{\gamma}^{2} \\
& \times\left[\mathcal{G}_{\sigma}\left(i \varepsilon_{n}+i \omega_{l}, \mathbf{k}+\mathbf{q}\right)\right]_{m m^{\prime}}\left[\mathcal{G}_{\sigma}\left(i \varepsilon_{n}, \mathbf{k}\right)\right]_{m^{\prime} m}
\end{aligned}
$$

is a a polarization function of quasi-particles and

$$
\tilde{P}_{\alpha}\left(i \omega_{l}\right)=\frac{1}{N} \sum_{\mathbf{q}} P_{\alpha}\left(i \omega_{l}, \mathbf{q}\right)
$$

is its local part. Because any single-site term is considered in the unperturbed state, the single-site part is subtracted in Eq. (3.52).

For the spin channel $(\alpha=s)$, Eqs. (3.53) and (3.54) become so simple as

$$
P_{s}\left(i \omega_{l}, \mathbf{q}\right)=\frac{1}{(2 l+1) N} \sum_{m \mathbf{k}} \frac{f\left[\xi_{m}(\mathbf{k}+\mathbf{q})\right]-f\left[\xi_{m}(\mathbf{k})\right]}{\xi_{m}(\mathbf{k})-\xi_{m}(\mathbf{k}+\mathbf{q})-i \omega_{l}}
$$

and

$$
\tilde{P}_{s}(0)=\int_{-\infty}^{\infty} d x \int_{-\infty}^{\infty} d y \frac{f(y)-f(x)}{x-y} \rho^{*}(x) \rho^{*}(y),
$$

with

$$
\rho^{*}(\epsilon)=\frac{1}{2 l+1} \sum_{m} \delta\left(\epsilon-\xi_{m}(\epsilon)\right)
$$

the density of quasi-particle states per spin and band. For orbital $(\alpha=o)$ and combined $(\alpha=s o)$ channels,

$$
\begin{gathered}
P_{\alpha}\left(i \omega_{l}, \mathbf{q}\right)=-\frac{2 l-1}{(2 l+1) N} \sum_{\mathbf{k}} \frac{f\left[\xi_{l}(\mathbf{k}+\mathbf{q})\right]-f\left[\xi_{l}(\mathbf{k})\right]}{\xi_{l}(\mathbf{k})-\xi_{l}(\mathbf{k}+\mathbf{q})-i \omega_{l}} \\
+\frac{8 l}{(2 l+1) N} \sum_{\mathbf{k}} \frac{f\left[\xi_{-l}(\mathbf{k}+\mathbf{q})\right]-f\left[\xi_{l}(\mathbf{k})\right]}{\xi_{l}(\mathbf{k})-\xi_{-l}(\mathbf{k}+\mathbf{q})-i \omega_{l}} \\
+\frac{2 l(2 l-1)}{(2 l+1) N} \sum_{\mathbf{k}} \frac{f\left[\xi_{-l}(\mathbf{k}+\mathbf{q})\right]-f\left[\xi_{-l}(\mathbf{k})\right]}{\xi_{-l}(\mathbf{k})-\xi_{-l}(\mathbf{k}+\mathbf{q})-i \omega_{l}}
\end{gathered}
$$

This exchange interaction includes no intrasite part so that $\sum_{\mathbf{q}} I_{\alpha}^{(Q)}\left(i \omega_{l}, \mathbf{q}\right)=0$. When $I_{\alpha}^{(Q)}(0,+0)<0$, $I_{\alpha}^{(Q)}(0, \mathbf{q})$ takes its maximum value at non-zero $\mathbf{q}$. In this paper, it is classified as being antiferromagnetic when $I_{\alpha}^{(Q)}(0,+0)<0$ and as being ferromagnetic when $I_{\alpha}^{(Q)}(0,+0)>0$. As was studied in the previous paper,25 the temperature dependence of $I_{\alpha}^{(Q)}(0, \mathbf{q})$ is responsible for the Curie-Weiss law. It is easy to see from Eq. (3.52) or (4.1) that $I_{\alpha}^{(Q)}(0, \mathbf{q})$ is of the order of $k_{B} T_{K}$.

\section{FLAT-BAND AND BAND-EDGE MODELS}

\section{A. Instability condition}

Assume $T=0 \mathrm{~K}$ in this section. Then, it follows that $P_{s}(0,|\mathbf{q}| \rightarrow+0)=\rho^{*}(0)$ and

$$
\frac{1}{4} I_{s}^{(Q)}(0,+0)=\frac{\tilde{\phi}_{s}}{\tilde{\phi}_{\gamma}}\left[1-\frac{\tilde{P}_{s}(0)}{\rho^{*}(0)}\right] \frac{1}{\tilde{\chi}_{s}^{*}(0)}
$$

Here, 
Note that $\rho^{*}(0)=\tilde{\phi}_{\gamma} \rho(0)$ to leading order in $1 / d$. An instability condition against ferromagnetism is simply given by $1-(1 / 4) I_{s}(0,0) \tilde{\chi}_{s}(0)<0$ or

$$
1-\frac{\tilde{\phi}_{s}}{\tilde{\phi}_{\gamma}}\left[1-\frac{\tilde{P}_{s}(0)}{\rho^{*}(0)}\right]-\Delta X_{s}<0,
$$

with

$$
\Delta X_{s}=\frac{1}{4}\left[I_{s}^{(s)}(0,0)-4 \Lambda_{s}(0,0)\right] \tilde{\chi}_{s}(0) .
$$

When $J$ is strong enough, the superexchange interaction, $I_{s}^{(s)}(0,0)$, is ferromagnetic, as is discussed in Sec. III B. The mode-mode coupling, $\Lambda_{s}(0,0)$, can be ignored in infinite dimensions $(d \rightarrow+\infty)$. It is likely that $\Delta X_{s}>0$ when $d, l$ and $J$ are large enough. When Eq. (4.3) is positively large enough, ferromagnetic instability occurs even when neither of the flat-band and band-edge conditions is satisfied. The SCR theory 2123 can only be applied to such ferromagnets in finite dimensions $(d \ll+\infty)$. Then, it is examined in this section whether or not ferromagnetic instability is possible even when $\Delta X_{s}$ is as small as $\left|\Delta X_{s}\right| \ll k_{B} T_{K}$ or $\Delta X_{s}=0$.

The signs of Eq. 4.1) or $\left[1-\tilde{P}_{s}(0) / \rho^{*}(0)\right]$ are functionals of $\rho^{*}(\epsilon)$. In order to examine the flat-band and band-edge conditions, we consider the following model of $\rho^{*}(\epsilon)$ : It is given by the sum of a narrow triangular one and a wide square one and the chemical potential is just at the peak of the triangle in such a way that

$$
\rho^{*}(\epsilon)=c \rho_{n}^{*}(\epsilon)+(1-c) \rho_{w}^{*}(\epsilon),
$$

where $\rho_{n}^{*}(\epsilon)$ and $\rho_{w}^{*}(\epsilon)$ are given by

$$
\rho_{n}^{*}(\epsilon)=\left\{\begin{array}{cl}
\frac{2}{w_{n}}\left(1-\left|\frac{\epsilon}{e_{n d}}\right|\right), & e_{n d} \leq \epsilon \leq 0 \\
\frac{2}{w_{n}}\left(1-\left|\frac{\epsilon}{e_{n u}}\right|\right), & 0 \leq \epsilon \leq e_{n u} \\
0, & \epsilon<e_{n d}, e_{n u}<\epsilon
\end{array},\right.
$$

with $w_{n}=e_{n u}-e_{n d}$, and

$$
\rho_{w}^{*}(\epsilon)=\left\{\begin{array}{cl}
\frac{1}{w_{w}}, & e_{w d} \leq \epsilon \leq e_{w u} \\
0, & \epsilon<e_{w d}, e_{w u}<\epsilon
\end{array}\right.
$$

with $w_{w}=e_{w u}-e_{w d}$. For the sake of simplicity, our examination is restricted to a realistic case, where a flat band lies within broad bands: $e_{n d}<0<e_{n u}, e_{w d}<0<$ $e_{w u}$, and $w_{w} \gg w_{n}$ so that

$$
\rho_{n}^{*}(0) \gg \rho_{w}^{*}(0) \text {. }
$$

It is also assumed that the quasi-particle density of states is normalized in such a way that

$$
\int_{-\infty}^{+\infty} d \epsilon \rho^{*}(\epsilon)=1
$$

Then, the carrier density per site is given by

$$
\begin{aligned}
n & =2(2 l+1) \int_{-\infty}^{+\infty} d \epsilon f(\epsilon) \rho^{*}(\epsilon) \\
& =2(2 l+1)\left[c \frac{\left|e_{n d}\right|}{w_{n}}+(1-c) \frac{\left|e_{w d}\right|}{w_{w}}\right] .
\end{aligned}
$$

Herer uttinger's sum rule or the Fermi-surface sum rule 10 11 is made use of.

In Eq. (4.4), $c$ is the weight of the triangular part. However, it is never a free parameter in this paper. When Eq. (2.6) is satisfied, our model is nothing but the flatband model. Such a flat-band model is simulated by $\rho^{*}(\epsilon)$ with $c \simeq 1 /(2 l+1)$. When interband transfer terms $E_{l-l}(\mathbf{k})$ are small, on the other hand, all the $2 l+1$ bands are degenerate. Such a model is simulated by $\rho^{*}(\epsilon)$ with $c=1$ or $c=0$.

It follows that

$$
\begin{aligned}
\tilde{P}_{s}(0)= & c^{2} \rho_{n}^{*}(0) A_{n}\left(\frac{\left|e_{n d}\right|}{w_{n}}\right)+(1-c)^{2} \rho_{w}^{*}(0) A_{w}\left(\frac{\left|e_{w d}\right|}{w_{w}}\right) \\
+c(1-c) \rho_{w}^{*}(0) & {\left[\frac{2 e_{n u}}{w_{n}} A_{n w}\left(\frac{\left|e_{w d}\right|}{e_{n u}}\right)+\right.} \\
& \left.+\frac{2\left|e_{n d}\right|}{w_{n}} A_{n w}\left(\frac{e_{w u}}{\left|e_{n d}\right|}\right)\right],
\end{aligned}
$$

with

$$
\begin{aligned}
A_{n}(x)=- & \frac{2}{3}\left\{1+\frac{x[x+3(1-x)]}{1-x} \ln x\right. \\
& \left.+\frac{(1-x)[(1-x)+3 x]}{x} \ln (1-x)\right\},
\end{aligned}
$$

$$
A_{w}(x)=-2[x \ln x+(1-x) \ln (1-x)],
$$

and

$$
A_{n w}(x)=\ln x+(x+1)^{2} \ln \left(1+\frac{1}{x}\right)-x .
$$

FIG. 4. A solid line shows $A_{n}(x)$, a dotted line $A_{w}(x)$, and a broken line $A_{n w}(x)$.

The instability condition (4.2) is more easily satisfied for smaller $\tilde{P}_{s}(0)$. Fig. 1 shows $A_{n}(x), A_{w}(x)$, and $A_{n w}(x)$. Note that $A_{n}(0)=A_{n}(1)=0 ; A_{w}(0)=$ $A_{w}(1)=0 ; A_{n w}(0)=0$ and $A_{n w}(x)$ increases only logarithmically with increasing $x$ in such a way that

$$
A_{n w}(x)=\ln x+\frac{3}{2}+\frac{1}{3 x}-\frac{1}{12 x^{2}}+\cdots
$$


for $x \gg 1$. When the chemical potential is around one of the band-edges, ferromagnetism is favored. On the other hand, $A_{n}(x)$ and $A_{w}(x)$ take their largest values at $x=1 / 2 ; A_{n}(1 / 2)=-(2 / 3)+(8 / 3) \ln 2=1.1873 \cdots$ and $A_{w}(1 / 2)=2 \ln 2=1.38629 \cdots$. When the chemical potential is around the band center, ferromagnetism is disfavored. This is one of the reasons why the band-edge condition is desired to be satisfied for itinerant-electron ferromagnetism.

\section{B. Flat-band model}

Consider the limit of $w_{w} / w_{n} \rightarrow+\infty$ or

$$
\rho_{w}^{*}(0) / \rho_{n}^{*}(0) \rightarrow+0
$$

in the case of nonzero $c(c>0)$. In this limit,

$$
\tilde{P}_{s}(0) / \rho^{*}(0)=c A_{n}\left(\left|e_{n d}\right| / w_{n}\right) .
$$

Note that $c \simeq 1 /(2 l+1)$ is smaller than unity. This is one of the reasons why ferromagnetism is favored in the flat-band model. Eq. (4.15) vanishes in the limit of

$$
l \rightarrow+\infty
$$

or

$$
\left|e_{n d}\right| / w_{n} \rightarrow+0 \text {. }
$$

Eq. (4.17) is nothing but the band-edge condition. Then, the instability condition (4.2) with $\Delta X_{s}=0$ becomes quite simple as

$$
1-\left(\tilde{\phi}_{s} / \tilde{\phi}_{\gamma}\right)<0
$$

In the Kondo problem, $\tilde{\phi}_{s} / \tilde{\phi}_{\gamma}$ is called the Wilson ratio. Call it the local Wilson ratio in this paper. In general, it is close to unity in the low density limit of $n \rightarrow+0$ and is larger than unity for $n>1$. The carrier density is simply given by

$$
n=2(2 l+1)\left(\left|e_{w d}\right| / w_{w}\right)
$$

When it is close to unity, $n \simeq 1$, charge fluctuations are substantially suppressed, so that $\tilde{\phi}_{c} \ll \tilde{\phi}_{s}$ and $\tilde{\phi}_{c} \ll \tilde{\phi}_{o}$, as is examined in Appendix A. Then, it follows from Eq. 3.3) that

$$
\tilde{\phi}_{\gamma} \simeq \frac{3 \tilde{\phi}_{s}}{2(2 l+3)}+\frac{4 l(l+1) \tilde{\phi}_{o}}{(2 l+1)(2 l+3)} .
$$

In the case of $U=U^{\prime}$ and $J=0$, it follows that $\tilde{\phi}_{s}=\tilde{\phi}_{o}$, so that the local Wilson ratio is as large as

$$
\frac{\tilde{\phi}_{s}}{\tilde{\phi}_{\gamma}}=1+\frac{5 l+3}{8 l^{2}+11 l+3}>1 .
$$

Here, $\tilde{\phi}_{c}=0$ is assumed. When $U>U^{\prime}$ or $J>0$, it follows that $\tilde{\phi}_{s}>\tilde{\phi}_{o}$. The local Wilson ratio is larger than Eq. (4.21) so that the instability condition, Eq. (4.2) or 4.18, is satisfied in such a case. As long as the Hund coupling is nonzero $(J>0)$, the ferromagnetic instability condition is satisfied in the flat-band model with large enough $l$ and $d$.

\section{Band-edge model}

Consider the low density limit of $n \rightarrow+0$ in the case of $c=1$ or $c=0$. According to the above argument, the chemical potential should be at the band bottom. It follows that $\tilde{P}_{s}(0) \rightarrow+0$ in the limit of $n \rightarrow+0$. The instability condition is also given by Eq. (4.18).

In the single-band model, $\lim _{n \rightarrow+0}\left(\tilde{\phi}_{s} / \tilde{\phi}_{\gamma}\right) \simeq 1$ but $\lim _{n \rightarrow+0}\left(\tilde{\phi}_{s} / \tilde{\phi}_{\gamma}\right)<1$ as is shown in Appendix A. The superexchange interaction is antiferromagnetic in general, and it vanishes in the limit of $U / k_{B} T_{k} \rightarrow+\infty$. The single-band model with $n \rightarrow 0$ and $U / k_{B} T_{K} \rightarrow+\infty$ is close to a quantum critical point for ferromagnetic instability. However, the instability condition is not satisfied.

In multi-band models, on the other hand, it follows that $\lim _{l \rightarrow+\infty} \lim _{n \rightarrow+0}\left(\tilde{\phi}_{s} / \tilde{\phi}_{\gamma}\right)=1$ for $J=0$ while $\lim _{l \rightarrow+\infty} \lim _{n \rightarrow+0}\left(\tilde{\phi}_{s} / \tilde{\phi}_{\gamma}\right)>1$ for $J>0$. The superexchange interaction is ferromagnetic for non-zero $J>0$ and large enough $l$. As long as the Hund coupling is nonzero $(J>0)$, therefore, the ferromagnetic instability condition Eq. (4.2) is generally satisfied in the limit of low densities $(n \rightarrow+0)$, large band multiplicity $(l \rightarrow+\infty)$, and large spatial dimensions $(d \rightarrow+\infty) 38$

\section{DISCUSSION}

In the single-band model, the superexchange interaction is antiferromagnetic and it vanishes in the large limit of $U$. The novel exchange interaction is antiferromagnetic for almost half filling, for example, according to Fig. 4. Therefore, the ground state must be antiferromagnetic or close to a antiferromagnetic critical point in the thermodynamic limit of small hole densities discussed in Introduction. Ferromagnetic instability cannot occur in this limit. Nagaoka and Thouless's ferromagnetism is a singular point as afunction of carrier numbers.

Kanamori's result 1 can also be explained within the framework of this paper. Both the flat-band and bandedge conditions are satisfied in Kanamori's model. Then, the novel exchange interaction is ferromagnetic so that the ground state must be a paramagnetic state close to a ferromagnetic quantum critical point. One can argue that ferromagnetic instability can occur in Kanamori's model if a weak ferromagnetic superexchange interaction is phenomenologically included; the superexchange interaction cannot be ferromagnetic in any single-band model.

In thefflat-band models considered by Mielke and Tasaki, 5 different orbits are located at different positions within a unit cell. In general, the Coulomb interaction between different positions is much weaker than the on-site one. Their models correspond to the case of $U>0, U^{\prime}=0$ and $J=0$ in Eq. (2.8) within the framework of this paper. When $U^{\prime}$ and $J$ are small or when $U^{\prime}=J$, the derivation of the superexchange interaction in this paper breaks down, as is implied by Eq. (3.44), where its denominators vanish. Once a large enough but still small $U^{\prime}\left(U^{\prime}>J\right)$ between different orbits is introduced, the formulation of this paper can be applied to such variant models of Mielke and Tasaki's. A strong ferromagnetic superexchange interaction exists in such models. This argument implies the existence of a strong ferromagnetic exchange interaction or an effective 
strong Hund coupling in the flat-band model of Mielke and Tasaki's, which corresponds to the ferromagnetic superexchange interaction considered in this paper. This is another reason why ferromagnetism is favored in the flat-band models.

Arguments on the flat-band condition in Sec. IV are restricted to a rather realistic case, where a flat band lies within broad bands. If the hybridization or mixing between flat and broad bands is strong as it is in our model, the treatment of this paper can be extended to the case where a flat band lies outside broad bands. Because of crystalline fields in cubic lattices, for example, $d$ orbitals split into $d \epsilon$ and $d \gamma$ multiplets. A model where only $d \epsilon$ or $d \gamma$ multiplet is taken into account corresponds to $l=1$ or $l=1 / 2$, while a model where all $d$ orbitals are taken into account corresponds to $l=2$. Although the superexchange interaction is definitely ferromagnetic for large l's, whether it is ferromagnetic or antiferromagnetic depends rather sensitively on $l$ in the region of small $l$ 's such as $l=1$ and $l=2$, as are shown in Figs. 2 and 3 . Not only orbitals around the chemical potential but also all other orbitals far from the chemical potential should be taken into account for a quantitative discussion on the magnitude of the superexchange interaction as well as its signs. It is interesting to extend the theory of this paper to treat such models with non-degenerate $d$ or $f$ orbitals and models without the symmetry of Eqs. (2.4) and (2.10). Such extensions seem to be indispensable to explain quantitatively itinerant-electron ferromagnetism in actual metals or alloys.

It is assumed in this paper that only electrons or holes exist. It is also interesting to extend the theory to treat ferromagnetism in semimetals, where electrons and holes coexist.

Because the superexchange was originally derived in insulating phases, it is controversial whether or not it exists in metallic phases. The derivation of this paper is based on Eq. (3.31) together with the Ward identity.35 It is obvious that the superexchange interaction exists even in metallic phases as long as the Mott-Hubbard splitting exists. When localized levels are so deep that $\epsilon_{a}-\mu \ll-k_{B} T_{K}$, Eq. (3.31) is a good approximation only for $\left|\varepsilon_{n}\right| \gg k_{B} T_{K}$. In this case, Gutzwiller's narrow quasi-particle band appear at the chemical potential between the lower and upper Hubbard bands. The system is a metal because of Gutzwiller's band. However, Gutzwiller's band plays no role in the process of the superexchange interaction. When localized levels are so shallow that $\epsilon_{a}-\mu \gtrsim-k_{B} T_{K}$, Eq. (3.31) is a rather good approximation even for the vicinity of the chemical potential; according to Eq. (3.31), the mass enhancement factor is $\tilde{\phi}_{\gamma}=1-n[2 l /(2 l+1)]$, which is consistent with Gutzwiller's result given by Eq. (1.3) in the large limit of $l \rightarrow+\infty$. Gutzwiller's band and the lower Hubbard band merge into a band and the upper Hubbard band lies above the merged band. In both the cases, the Mott-Hubbard splitting exists so that the superexchange interaction exists.

The superexchange interaction in metallic phases can also be understood by the following argument. Because the typical time scale of quasi-particles, which is $O\left(\hbar / k_{B} T_{B}\right)$, is much longer than that of pair excitations of electrons or holes across the Mott-Hubbard gap, which is $O(\hbar / U)$ or $O\left(\hbar /\left(U^{\prime} \pm J\right)\right)$, all the exchange processes can be finished while a pair of quasi-particles meet occasionally and part again. As long as the Mott-Hubbard splitting exists and Eq. (3.26) is satisfied, therefore, the superexchange interaction works between quasi-particle pairs that occasionally come close to each other.

The novel exchange interaction considered in Sec. III is similar to the double and the RKKY exchange interactions, each of which arises from that of pair excitations of conduction electrons. The double and the RKKY exchange interactions are essentially the same; in many cases the double exchange interaction seems to stand for the $\mathbf{q}=0$ component of the RKKY exchange interaction. However, the novel exchange interaction is totally different from the double or RKKY exchange interaction. When the RKKY or double exchange interaction is derived, the existence of localized spins are assumed. The double or RKKY exchange interaction is an exchange interaction working between localized spins in metals. On the other hand, the novel exchange interaction works between itinerant quasiparticles. The strength of the RKKY or double interactions is inversely proportional to the width of conduction bands, while the strength of the novel exchange interaction is proportional to the bandwidth of quasiparticles.

Because the local or intrasite part of the RKKY or double exchange interaction can play no role, its intrasite part should be subtracted. Then, whether the RKKY or double exchange interaction is ferromagnetic or antiferromagnetic depends on the position of the chemical potential. The band-edge condition also applies to this case. When the chemical potential is at the top or bottom region of conduction bands, it is ferromagnetic. When the chemical potential lies in the center region of conduction bands or the nesting of the Fermi surface is significant, it is antiferromagnetic. The situation is similar to that for the novel exchange interaction.

The double or RKKY exchange interaction arises from the second-order perturbation in the $s$ - $d$ exchange. When the $s$ - $d$ exchange interaction is ferromagnetic, the volume of the Fermi surface is simply given by the number of conduction electrons. In the band-edge model, the RKKY exchange interaction is ferromagnetic and metallic ferromagnetism is possible. Ferromagnetism caused by this type of the double or RKKY exchange interaction is nothing but ferromagnetism examined by Zener. 39 It should never be called itinerant-electron ferromagnetism, but it is local-moment ferromagnetism in metals.

When the $s$ - $d$ exchange interaction is antiferromagnetic, localized spins must be screened by the Kondo effect. The analogy between the periodic antiferromagnetic $s-d$ model and the periodic Anderson model implies that when higher-order processes in the $s$ - $d$ exchange interaction are considered the double or RKKY exchange interaction should be replaced by a similar one to the novel exchange interaction studied in this paper. The analogy also implies that the volume of the Fermi surface must be given by the number of total electrons or by the sum of those of conduction electrons and localized spins. When the volume of electron or hole Fermi surface is small, this exchange interaction must be ferromagnetic. For example, assume that there is only a single conduction band. Denote the average number of conduction electrons per site by $n_{c}$. When the number of localized and screened spins is taken into account, $n_{c}=1$ corresponds to the 
total electron number 2 per site. This exchange interaction must be ferromagnetic for $n_{c} \simeq 1$, because the band edge condition is satisfied. We speculate that if the critical temperature is much lower than the Kondo temperature ferromagnetism caused by this exchange interaction must be physically the same as itinerant-electron ferromagnetism studied in this paper 40

\section{SUMMARY}

Itinerant-electron ferromagnetism in multi-band models is examined within a theoretical framework of the competition between the two leading-order effects in $1 / d$ : magnetic instability caused by Weiss' mean fields or exchange interactions and the quenching of magnetic moments by the Kondo effect.

Two exchange interactions are responsible for ferromagnetic instability. One is the superexchange interaction, which arises from the virtual exchange of pair excitations of electrons or holes across the Mott-Hubbard gap. When the Hund coupling is strong enough and the band multiplicity is large enough, the superexchange interaction becomes ferromagnetic. The other is the novel exchange interaction arising from the virtual exchange of pair excitations of quasi-particles, which is also responsible for the Curie-Weiss law. Its strength is proportional to the bandwidth of quasi-particles, and its signs depend on the shape of the density of quasiparticle states and the position of the chemical potential. This exchange interaction is ferromagnetic, when the flat-band condition is satisfied so that the density of states has a sharp peak around the chemical potential within or outside a broad band or broad bands or when the band-edge condition is satisfied so that the chemical potential is at one of the band edges. When the electron or hole density is close to unity per unit cell, charge fluctuations are substantially suppressed so that the local Wilson ratio is enhanced. Because spin fluctuations are more enhanced than orbital fluctuations in the presence of the Hund coupling, the local Wilson ratio is also enhanced. The enhancement of the local Wilson ratio leads to the enhancement of the novel exchange interaction. When the sum of the superexchange and the novel exchange interactions overcomes the quenching of magnetic moments or the suppression of magnetic instability by local and intersite spin fluctuations, a ferromagnetic state is stabilized at low enough temperatures.

In conclusion, the following three conditions should be satisfied in order that a ferromagnetic state is stabilized at low enough temperatures: (i) There are two or more than two bands, (ii) the on-site Hubbard repulsion is much larger than the energy scale of local quantum spin fluctuations, and (iii) the Hund coupling is strong enough. Some or all of the following conditions should also be satisfied: (iv) There is an almost dispersionless band within broad ones, (v) the chemical potential is at the top or bottom of bands, and (vi) the electron or hole density is close to unity per unit cell.

\section{ACKNOWLEDGMENTS}

entific Research (C) No. 13640342 from the Ministry of Education, Cultures, Sports, Science and Technology of Japan.

\section{APPENDIX A: NON-MAPPED SINGLE-IMPURITY ANDERSON MODEL}

Consider $\mathcal{H}_{A}$ given by Eq. (2.13). In this Appendix, it is assumed that the hybridization energy defined by

$$
\Delta=\frac{1}{N} \sum_{\mathbf{k}}\left|v_{m \mathbf{k}}\right|^{2} \delta\left(\epsilon+\mu-e_{c}(\mathbf{k})\right)
$$

does not depend on $\epsilon$ as well as $m$ and the conduction bandwidth $2 D$ is much larger than $U, U^{\prime}$ and $J$. When $U=U^{\prime}=J=0$, the density of states of localized electrons is given by

$$
\rho_{0}(\epsilon)=\frac{1}{\pi} \frac{\Delta}{\left(\epsilon-\epsilon_{a}+\mu\right)^{2}+\Delta^{2}} .
$$

When the Friedel sum rule or the Fermi-liquid relation 41 is made use of, the density of states at the chemical potential for non-zero $U, U^{\prime}$ and $J$ is simply given by

$$
\rho(0)=\frac{1}{\pi \Delta} \sin ^{2}\left(\frac{\pi n}{2(2 l+1)}\right)
$$

as a function of $n=\sum_{m \sigma}\left\langle n_{m \sigma}\right\rangle$. Our study in this Appendix is also restricted to $n<1$.

Introduce infinitesimally small external fields given by Eq. (3.1) with $H_{s o}=0$, and define

$$
\epsilon_{m \sigma}=\epsilon_{a}-\Delta \mu-\frac{1}{2} \sigma g_{s} \mu_{B} H_{s}-m g_{o} \mu_{B} H_{o} .
$$

The ground state wave function, $\Phi$, is expanded in such a way that 31

$$
\begin{gathered}
\Phi=\left[\Gamma_{0}+\sum_{m \mathbf{k} \sigma} \Gamma_{(m \sigma) ;(\mathbf{k} \sigma)} a_{\sigma \tau}^{\dagger} c_{\mathbf{k} \sigma}+\sum_{\mathbf{k} \mathbf{p} \sigma} \Gamma_{(\mathbf{k} \sigma) ;(\mathbf{p} \sigma)} c_{\mathbf{k} \sigma}^{\dagger} c_{\mathbf{p} \sigma}\right. \\
+\sum_{m \mathbf{k} \sigma} \sum_{m^{\prime} \mathbf{p} \sigma^{\prime}} \Gamma_{\left[(m \sigma)\left(m^{\prime} \sigma^{\prime}\right) ;\left(\mathbf{p} \sigma^{\prime}\right)(\mathbf{k} \sigma)\right]} \times \\
\left.\left.\times a_{m \sigma}^{\dagger} a_{m^{\prime} \sigma^{\prime}}^{\dagger} c_{\mathbf{p} \sigma^{\prime}} c_{\mathbf{k} \sigma}+\cdots\right]|0\rangle, \quad \text { (A } 5\right)
\end{gathered}
$$

with $|0\rangle$ the Fermi vacuum, and it satisfies

$$
\left[E-\mathcal{H}_{A}-\mathcal{H}_{\text {ext }}\right] \Phi=0 .
$$

In Eq. A5), $\Gamma_{\left[(m \sigma)\left(m^{\prime} \sigma^{\prime}\right) ;\left(\mathbf{p} \sigma^{\prime}\right)(\mathbf{k} \sigma)\right]}$ is an antisymmetrized coefficient defined in such a way that

$$
\begin{aligned}
& \Gamma_{\left[(m \sigma)\left(m^{\prime} \sigma^{\prime}\right) ;\left(\mathbf{p} \sigma^{\prime}\right)(\mathbf{k} \sigma)\right]}= \\
& \quad=\Gamma_{(m \sigma)\left(m^{\prime} \sigma^{\prime}\right) ;\left(\mathbf{p} \sigma^{\prime}\right)(\mathbf{k} \sigma)}-\Gamma_{\left(m^{\prime} \sigma^{\prime}\right)(m \sigma) ;\left(\mathbf{p} \sigma^{\prime}\right)(\mathbf{k} \sigma)} \\
& \quad-\Gamma_{(m \sigma)\left(m^{\prime} \sigma^{\prime}\right) ;(\mathbf{k} \sigma)\left(\mathbf{p} \sigma^{\prime}\right)}+\Gamma_{\left(m^{\prime} \sigma^{\prime}\right)(m \sigma) ;(\mathbf{k} \sigma)\left(\mathbf{p} \sigma^{\prime}\right)} .
\end{aligned}
$$

Eqs. (A5) and (A6) give a set of equations for $\Gamma$ 's:

$$
E \Gamma_{0}-\frac{1}{\sqrt{N}} \sum_{m \mathbf{k} \sigma} v_{\mathbf{k} \tau}^{*} \Gamma_{(m \sigma) ;(\mathbf{k} \sigma)}=0
$$




$$
\begin{array}{rrr}
{\left[E-\epsilon_{m \sigma}+e_{c}(\mathbf{k})\right] \Gamma_{(m \sigma) ;(\mathbf{k} \sigma)}-\frac{1}{\sqrt{N}} v_{m \mathbf{k}} \Gamma_{0}-} & \Delta E_{0}= & -\frac{\Delta}{\pi}\left[\ln \frac{D}{\Delta}+2 \ln \frac{D+U}{U}\right. \\
-\frac{1}{\sqrt{N}} \sum_{\mathbf{p}} v_{m \mathbf{p}} \Gamma_{(\mathbf{p} \sigma) ;(\mathbf{k} \sigma)} & \left.+3 l \ln \frac{D+U^{\prime}-J}{U^{\prime}-J}+l \ln \frac{D+U^{\prime}+J}{U^{\prime}+J}\right],
\end{array}
$$$$
-\frac{2}{\sqrt{N}} \sum_{m^{\prime} \mathbf{p} \sigma^{\prime}} v_{m^{\prime} \mathbf{p}}^{*} \Gamma_{\left[(m \sigma)\left(m^{\prime} \sigma^{\prime}\right) ;\left(\mathbf{p} \sigma^{\prime}\right)(\mathbf{k} \sigma)\right]}=0,
$$$$
\left[E-e_{c}(\mathbf{p})+e_{c}(\mathbf{k})\right] \Gamma_{(\mathbf{p} \sigma) ;(\mathbf{k} \sigma)}+
$$$$
-\frac{v_{m \mathbf{p}}^{*}}{\sqrt{N}} \Gamma_{(m \sigma) ;(\mathbf{k} \sigma)}+\cdots=0,
$$$$
\Gamma_{[(m \sigma)(m \sigma) ;(\mathbf{p} \sigma)(\mathbf{k} \sigma)]}=0,
$$$$
\left[E-\epsilon_{m \sigma}-\epsilon_{m-\sigma}-U+\right.
$$$$
\left.+e_{c}(\mathbf{k})+e_{c}(\mathbf{p})\right] \Gamma_{[(m \sigma)(m-\sigma) ;(\mathbf{p}-\sigma)(\mathbf{k} \sigma)]}-
$$$$
-\frac{v_{m \mathbf{p}}}{\sqrt{N}} \Gamma_{(m \sigma) ;(\mathbf{k} \sigma)}-\frac{v_{m \mathbf{k}}}{\sqrt{N}} \Gamma_{(m-\sigma) ;(\mathbf{p}-\sigma)}+\cdots=0,
$$$$
\left[E-\epsilon_{m \sigma}-\epsilon_{m^{\prime} \sigma}-U^{\prime}+J+\right.
$$$$
\left.+e_{c}(\mathbf{k})+e_{c}(\mathbf{p})\right] \Gamma_{\left[(m \sigma)\left(m^{\prime} \sigma\right) ;(\mathbf{p} \sigma)(\mathbf{k} \sigma)\right]}-
$$$$
-\frac{v_{m^{\prime} \mathbf{p}}}{\sqrt{N}} \Gamma_{(m \sigma) ;(\mathbf{k} \sigma)}-\frac{v_{m \mathbf{k}}}{\sqrt{N}} \Gamma_{\left(m^{\prime} \sigma\right) ;(\mathbf{p} \sigma)}+\cdots=0
$$$$
\left[E-\epsilon_{m \sigma}-\epsilon_{m^{\prime}-\sigma}-U^{\prime}+\right.
$$$$
\left.+e_{c}(\mathbf{k})+e_{c}(\mathbf{p})\right] \Gamma_{\left[(m \sigma)\left(m^{\prime}-\sigma\right) ;(\mathbf{p}-\sigma)(\mathbf{k} \sigma)\right]}+
$$$$
+J \Gamma_{\left[(m-\sigma)\left(m^{\prime} \sigma\right) ;(\mathbf{p}-\sigma)(\mathbf{k} \sigma)\right]}
$$$$
-\frac{v_{m^{\prime} \mathbf{p}}}{\sqrt{N}} \Gamma_{(m \sigma) ;(\mathbf{k} \sigma)}-\frac{v_{m \mathbf{k}}}{\sqrt{N}} \Gamma_{\left(m^{\prime}-\sigma\right) ;(\mathbf{p}-\sigma)}+\cdots=0,
$$

$$
\begin{aligned}
& {\left[E-\epsilon_{m-\sigma}-\epsilon_{m^{\prime} \sigma}-U^{\prime}+\right.} \\
& \left.+e_{c}(\mathbf{k})+e_{c}(\mathbf{p})\right] \Gamma_{\left[(m-\sigma)\left(m^{\prime} \sigma\right) ;(\mathbf{p}-\sigma)(\mathbf{k} \sigma)\right]}+ \\
& +J \Gamma_{\left[(m \sigma)\left(m^{\prime}-\sigma\right) ;(\mathbf{p}-\sigma)(\mathbf{k} \sigma)\right]}+\cdots=0, \quad(\mathrm{~A} 8 \mathrm{~h})
\end{aligned}
$$

and so on. In Eqs. (A8f), $\mathrm{A} 8 \mathrm{~g}$ ) and (A8h), $m \neq m^{\prime}$.

When small irrelevant terms are ignored, it follows from Eqs. (A8a)- A8h that

$$
\frac{1}{F(E)}=\frac{\Delta}{\pi} \sum_{m \sigma} \ln \left|\frac{D}{E-\Delta E_{0}-\epsilon_{m \sigma}^{*}+\mu}\right|,
$$

with

$$
\begin{aligned}
F(E)= & \frac{1}{-E}+\frac{1}{2 l+1}\left[\frac{1}{U+\epsilon_{a}-\mu}+\right. \\
& \left.+\frac{3 l}{U^{\prime}-J+\epsilon_{a}-\mu}+\frac{l}{U^{\prime}+J+\epsilon_{a}-\mu}\right],
\end{aligned}
$$

and

$$
\epsilon_{m \sigma}^{*}=\epsilon_{a}-g_{c}^{*} \Delta \mu-\frac{1}{2} \sigma g_{s}^{*} \mu_{B} H_{s}-m g_{o}^{*} \mu_{B} H_{o} .
$$

Here, $g_{c}^{*}, g_{s}^{*}$ and $g_{o}^{*}$ are renormalized $g$ factors:

$$
g_{c}^{*}=1+\frac{1}{2 l+1}\left[R(U)+3 l R\left(U^{\prime}-J\right)+l R\left(U^{\prime}+J\right)\right],
$$

$$
\frac{g_{s}^{*}}{g_{s}}=1+\frac{2 l}{2 l+1} R\left(U^{\prime}-J\right),
$$

and

$$
\begin{aligned}
\frac{g_{o}^{*}}{g_{o}}= & +\frac{1}{2 l+1} R(U) \\
& +\frac{2 l-1}{2 l+1}\left[\frac{3}{4} R\left(U^{\prime}-J\right)+\frac{1}{4} R\left(U^{\prime}+J\right)\right],
\end{aligned}
$$

with

$$
R(x)=\frac{4(2 l+1) \Delta}{\pi}\left[\frac{1}{x+\epsilon_{a}-\mu}-\frac{1}{D+x+\epsilon_{a}-\mu}\right] .
$$

Eq. A15) should be discarded for $l=0$. Note that $g_{c}^{*}>$ $g_{s}^{*} / g_{s} \geq g_{o}^{*} / g_{o}>1 ; g_{s}^{*} / g_{s}=g_{o}^{*} / g_{o}$ when $U=U^{\prime}$ and $J=0$, while $g_{s}^{*} / g_{s}>g_{o}^{*} / g_{o}$ when $U>U^{\prime}$ or $J>0$. Even in the absence of the spin-orbit interaction, the $g$ factor in the spin channel is enhanced in multi-band models.

When $\left\langle n_{m \sigma}\right\rangle \ll 1, F(E)$ is approximated by $F(E) \simeq$ $-1 / E$. The electron number is given by $n=-\partial E / \partial \Delta \mu$. The charge susceptibility is calculated so that

$$
\tilde{\chi}_{c}^{*}(0)=\frac{\partial n}{\partial \Delta \mu}=\left(g_{c}^{*}\right)^{2} \frac{\pi}{2(2 l+1) \Delta} n^{2}\left(g_{c}^{*}-n\right)
$$

as a function of $n$. In similar manners, spin and orbital susceptibilities are calculated so that

$$
\tilde{\chi}_{\alpha}^{*}(0)=\left(\frac{g_{\alpha}^{*}}{g_{\alpha}}\right)^{2} \frac{\pi}{2(2 l+1) \Delta} \frac{n^{2}}{g_{c}^{*}-n} .
$$

The expansion coefficients appearing in the single-site selfenergy are given by

$$
\tilde{\phi}_{c}=\left(g_{c}^{*}\right)^{2}\left(g_{c}^{*}-n\right) \frac{\left\langle\pi n_{m \sigma}\right\rangle^{2}}{\sin ^{2}\left\langle\pi n_{m \sigma}\right\rangle}
$$

for the charge channel, and

$$
\tilde{\phi}_{\alpha}=\left(\frac{g_{\alpha}^{*}}{g_{\alpha}}\right)^{2} \frac{1}{g_{c}^{*}-n} \frac{\left\langle\pi n_{m \sigma}\right\rangle^{2}}{\sin ^{2}\left\langle\pi n_{m \sigma}\right\rangle}
$$

for spin $(\alpha=s)$ and orbital $(\alpha=o)$ channels. Note that $\tilde{\phi}_{c}>\tilde{\phi}_{s} \gtrsim \tilde{\phi}_{o}>1$ in the low density limit of $n \rightarrow+0$ although $\tilde{\phi}_{s} \geq \tilde{\phi}_{o} \gg 1 \gg \tilde{\phi}_{c}$ for $n \simeq 1$. 
${ }^{1}$ J. Kanamori, Prog. Theor, Phys. 30, 275 (1963).

${ }^{2}$ A. Mielke, J. Phys. A24, 3311 (1991); ibid. A25, 4335 (1992); Phys. Lett. A174, 443 (1993);

${ }^{3}$ A. Mielke and H. Tasaki, Commun. math. phys. 158, 341 (1993).

${ }^{4}$ H. Tasaki, Phys. Rev. Lett. 69, 1608 (1992); ibid. 73, 1158 (1994); ibid. 74, 4678 (1995).

${ }^{5}$ K. Kusakabe and A. Aoki, Phys. Rev. Lett. 72, 144 (1994).

${ }^{6}$ Y. Nagaoka, Phys. Rev. 147, 392 (1966).

${ }^{7}$ D. J. Thouless, Proc. Phys. Soc. (London) A 86, 893 (1965).

8 J. Hubbard, Proc. Roy. Soc. A276, 238 (1963); ibid. A281, 401 (1964).

${ }^{9}$ M. C. Gutzwiller, Phys. Rev. Lett. 10, 159 (1963); Phys. Rev. 134, A293 (1963); ibid. A137, 1726 (1965).

${ }^{10}$ J. M. Luttinger and J. C. Ward, Phys. Rev. 118, 1417 (1960).

11 J. M. Luttinger, Phys. Rev. 119, 1153 (1960).

12 F. J. Ohkawa, Phys. Rev. B 44, 6812 (1991); J. Phys. Soc. Jpn. 60, 3218 (1991); ibid. 61, !615 (1991).

${ }^{13}$ K. G. Wilson, Rev. Mod. Phys. 47, 773 (1975).

${ }^{14}$ P. Nozières, J. Low. Temp. Phys. 17, 31 (1974).

${ }^{15}$ K. Yamada, Prog. Theor. Phys. 53, 970 (1975); ibid 54, 316 (1975).

${ }^{16}$ K. Yosida and K. Yamada, Prog. Theor. Phys. 53, 1286 (1970).

${ }^{17}$ For reviews see, e.g., N. Andrei, K. Furuya, and J. H. Lowenstein, Rev. Mod. Phys. 55, 331 (1983); A. M. Tsvelick and P. B. Wiegmann, Adv. Phys. 32, 453 (1983); A. Okiji and N. Kawakami, Springer Series in Solid State Science (Springer, Berlin, 1988), Vol. 77, p 63; P. Schlottmann, Phys. Rep. 118, 1 (1989).

${ }^{18}$ K. Yosida, Phys. Rev. 147 (1966) 223.

${ }^{19}$ W. Metzner and D. Vollhardt, Phys. Rev. Lett. 62, 324 (1989).

20 The Curie-Weiss law of local-moment magnets arises from the temperature dependence of local thermal spin fluctuations. In magnets, there are only two relevant leading order effects in $1 / d$ : local spin fluctuations and magnetic Weiss' mean fields. Local charge fluctuations and isotropic BCS superconducting fluctuations and their corresponding Weiss' mean fields are also of leading order in $1 / d$, but they are irrelevant in magnets.

${ }^{21}$ K. K. Murata and S. Doniach, Phys. Rev. Lett. 29, 285 (1972).

22 T. Moriya and A. Kawabata, J. Phys. soc. Jpn. 34, 639 (1973); ibid. 35, 669 (1973).

23 T. Moriya, Spin Fluctuations in Itinerant Electron Magnetism, Springer SEries in Solid-State Sciences 56, Springer-Verlag, Berlin Heidelberg New York Tokyo, 1985.

${ }^{24}$ F. J. Ohkawa, J. Phys. Soc. Jpn. 67, 525 (1998).

${ }^{25}$ E. Miyai and F. J. Ohkawa, Phys. Rev. B 61, 1357 (2000).

${ }^{26}$ L. Puech, J. M. Mignot, P. Lejay, P. Haen, J. Flouquet, and J. Voiron, J. Low. Temp. Phys. 70, 237 (1988).

27 J.-M. Mignot, J. Flouquet, P. Haen, F. Lapierre, L. Puech, and J. Voiron, J. Magn. Magn. \& Mater. 76\&77, 97 (1988).

${ }^{28}$ H. Satoh and F. J. Ohkawa, Phys. Rev. B 57, 5891 (1998); ibid. B 63, 184401 (2001).

${ }^{29}$ When no interband terms are included, the formulation of this paper can be applied to single-band models.

30 This relation can be only approximately satisfied in actual systems.

${ }^{31}$ In the large limit of $l, g_{o}$ and $g_{\text {so }}$ should be defined in such a way that they include a factor of $1 / \sqrt{l(l+1)}$. In a similar manner, $v_{m \mathbf{k}}$ should be defined in such a way that it includes $1 / \sqrt{2 l+1}$, so that $\pi \Delta \propto 1 /(2 l+1)$. Then, the perturbative scheme used in Appendix is nothing but the
$1 /(2 l+1)$ expansion.

${ }^{32}$ A. Yoshimori, Prog. Theor. Phys. 55, 67 (1976).

${ }^{33}$ L. M. Roth, Phys. Rev. 149, 306 (1966). When the interaction energy of two atoms due to admixture of ionic states was examined, the splitting between the two $S_{z}=0$ states of $S=1$ and $S=0$ due to the Hund coupling was not considered in this reference, as was shown in Table I there. Because the $S=1$ state gains a larger energy than the $S=0$ one, this splitting plays a critical role in deriving the ferromagnetic superexchange interaction, as is shown in Eq. (3.44) of this paper.

${ }^{34}$ In this case, the mapped Anderson model lies in the so called valence fluctuation regime. The Kondo temperature or energy can be still defined as the energy scale of local quantum spin fluctuations by Eq. (3.10).

35 J. C. Ward, Phys. Rev. 78, 182 (1950).

${ }^{36}$ In large spatial dimensions $(d \gg 1), t_{m i, m^{\prime} j}$ should be defined in such a way that it includes a factor of $1 / d^{\left(L_{i j} / 2\right)}$, with $L_{i j}$ the metropolitan distance between the $i$ th and $j$ th sites, 19 so that $I_{\alpha ; i j}^{(s)} \propto 1 / d^{L_{i j}}$ is of higher order in $1 / d$. Only when the contributions from neighboring sites are coherent, $I_{\alpha}^{(s)}\left(i \omega_{l}, \mathbf{q}\right)$ is of leading order in $1 / d$ for particular and discrete q's. It is of higher order for almost all q's. The $1 / d$ dependence of $I_{\alpha}^{(Q)}\left(i \omega_{l}, \mathbf{q}\right)$ and $I_{\alpha}^{(s Q)}\left(i \omega_{l}, \mathbf{q}\right)$ is similar to that of $I_{\alpha}^{(s)}\left(i \omega_{l}, \mathbf{q}\right)$.

${ }^{37}$ Intraband contributions $\left(r=r^{\prime}\right)$ are never significant, unless they are in the vicinity of the chemical potential.

${ }^{38}$ When $\rho^{*}(x)$ is of a Lorentzian shape in such a way that $\rho^{*}(x)=\left(\Delta^{*} / \pi\right) /\left[\left(x-\epsilon_{a}^{*}\right)^{2}+\left(\Delta^{*}\right)^{2}\right]$, the novel exchange interaction vanishes for any $\epsilon_{a}^{*}$ at $T=0 \mathrm{~K}$ because

$$
\int_{-\infty}^{+\infty} d x \int_{-\infty}^{+\infty} d y \frac{f(x)-f(y)}{y-x} \rho^{*}(x) \rho^{*}(y)=\rho^{*}(0) .
$$

${ }^{39}$ C. Zener, Phys. Rev. 81, 440 (1951).

${ }^{40}$ If $T \gg T_{K}$, localized spins are not screened. Then, the double or RKKY exchange interaction must be relevant for $T \gg T_{K}$, and it is presumably antiferromagnetic because the volume of the Fermi surface is large for $n_{c} \simeq 1$. A ferromagnetic state can never be stabilized above $T_{K}$ for $n_{c} \simeq 1$.

${ }^{41}$ H. Shiba, Prog. Theor. Phys. 54, 967 (1975). 

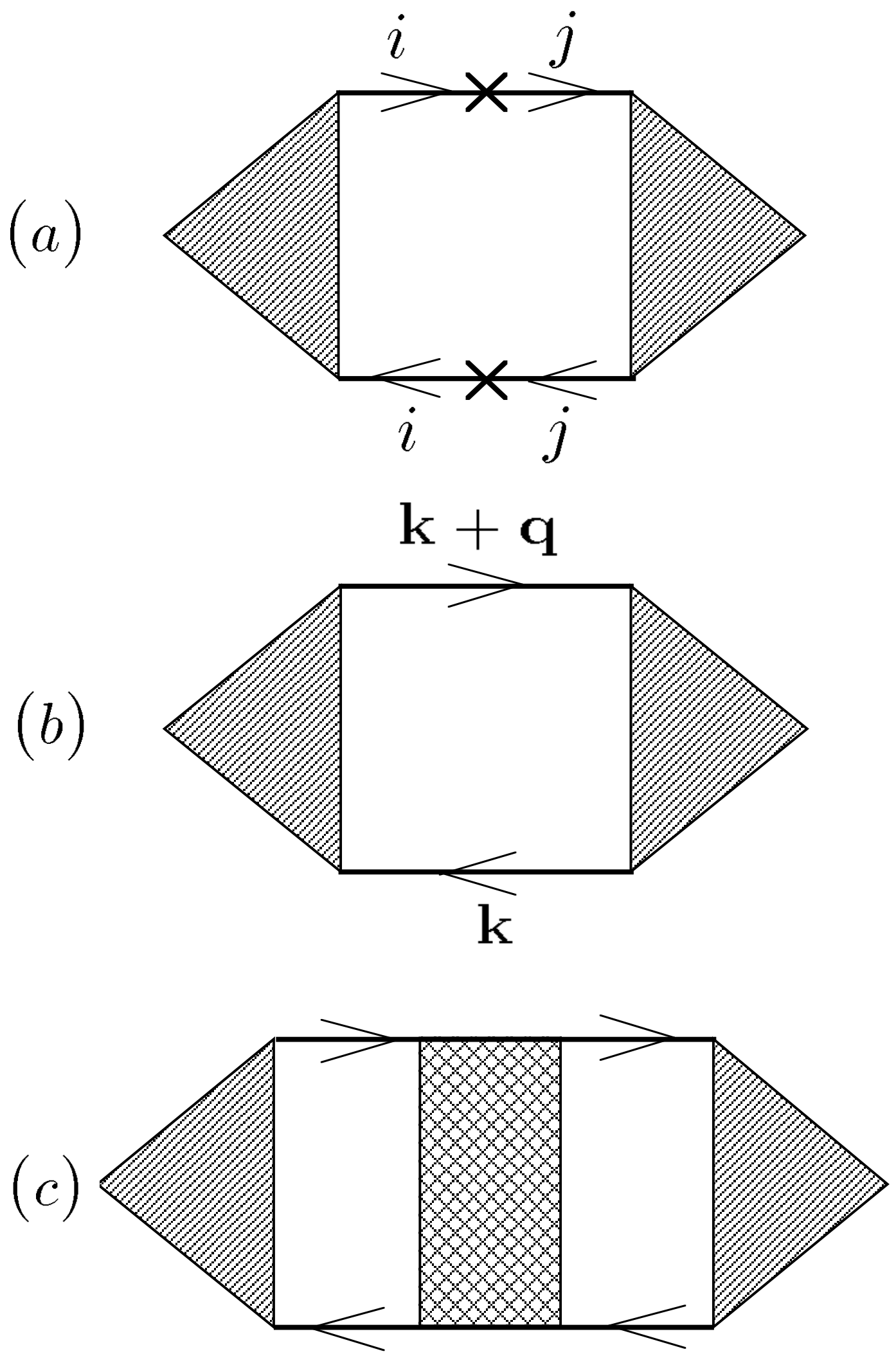

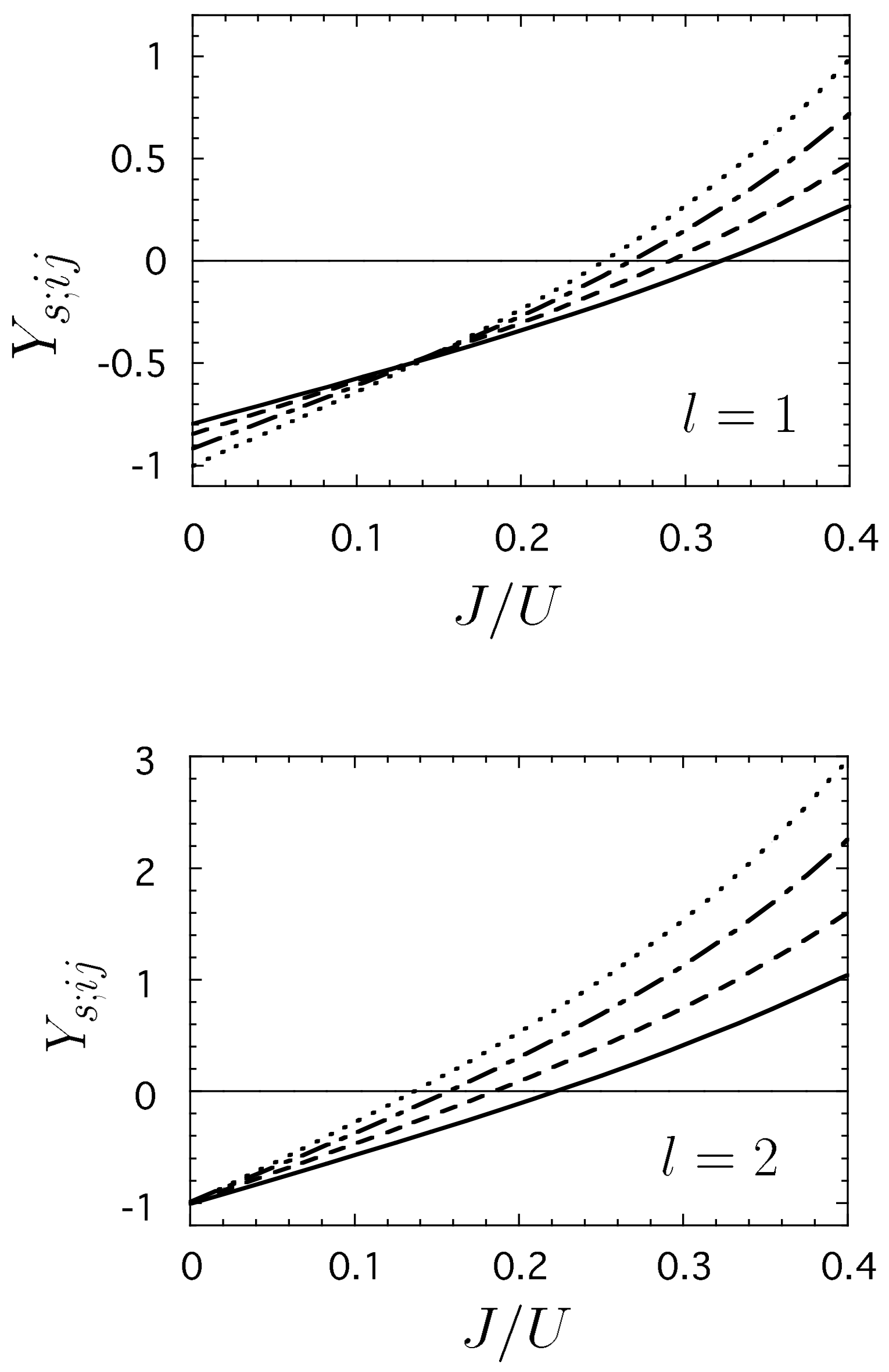

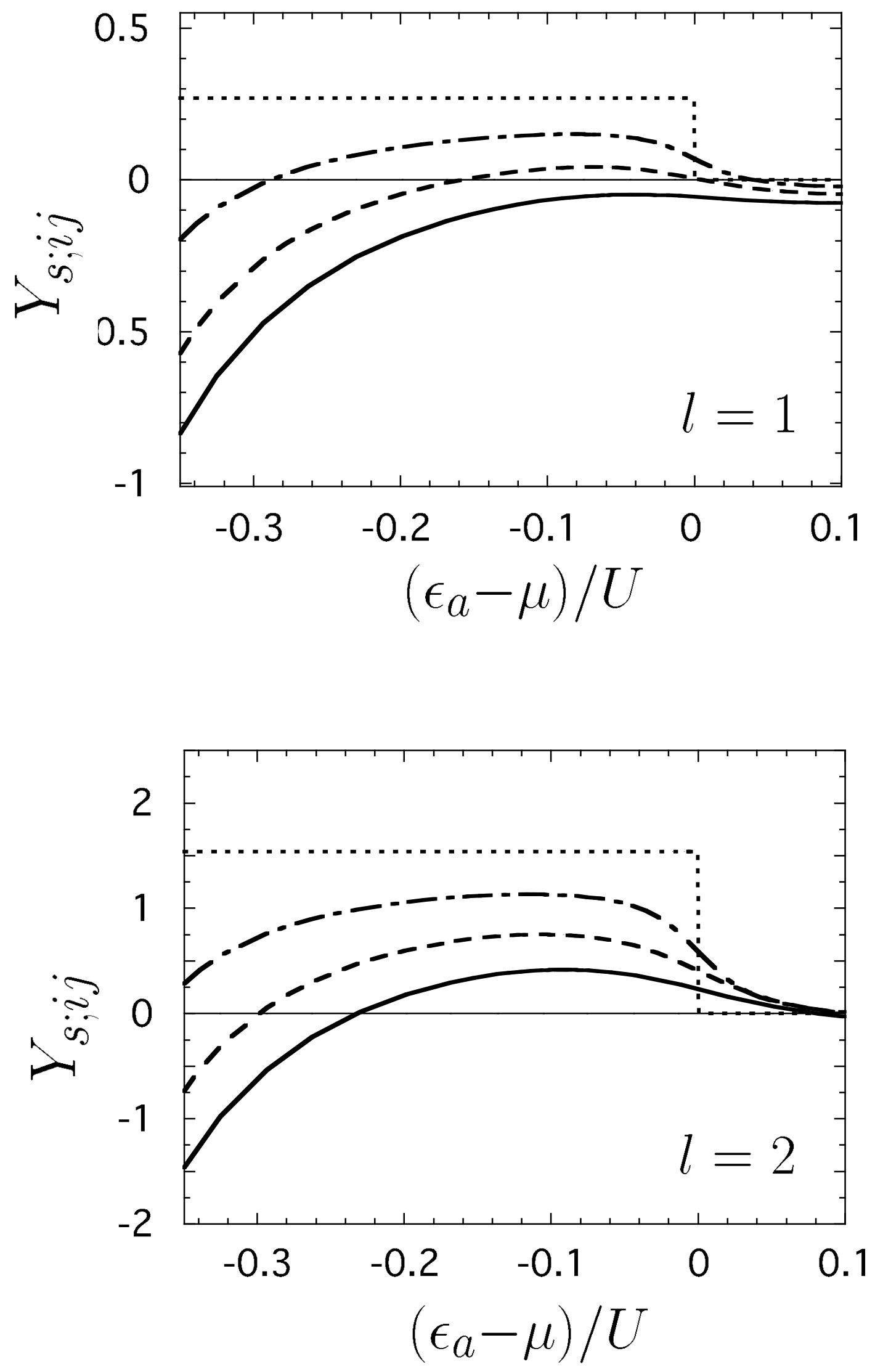


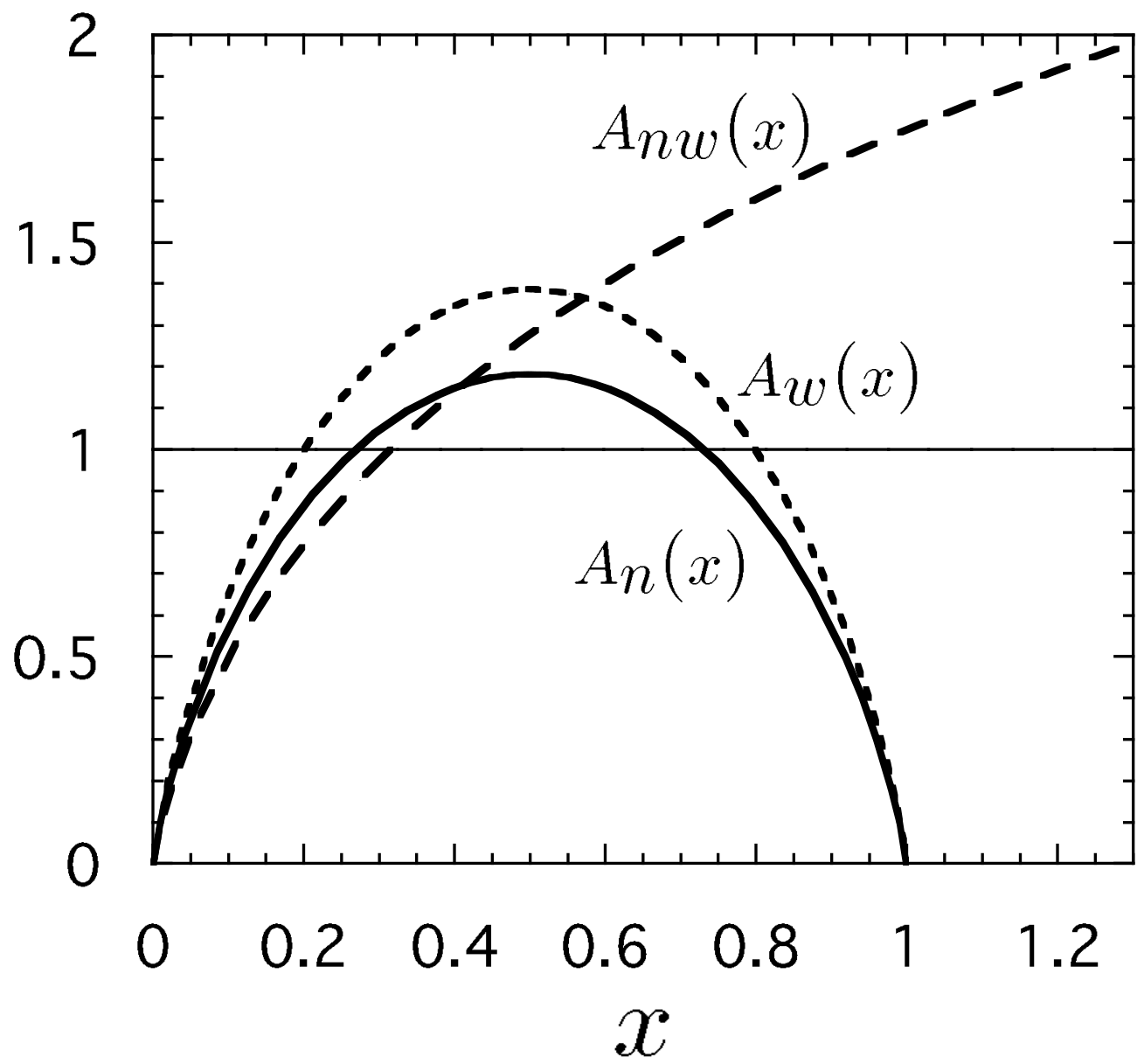

